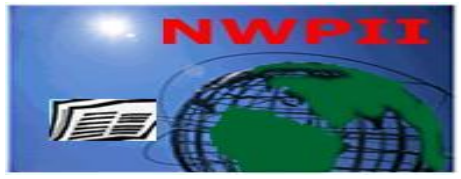

American Journal of

Biomedical Sciences

ISSN: 1937-9080

nwpii.com/ajbms

\title{
The Effect of Graviola Leaves Extract on the Progression of Pancreatic Cancer in Rats
}

\author{
Rana HM. Khafaga, Suzan M. Abdel-Tawab, Nagwa M. Assem, \\ Magda A. Meghad, \& Ahmad R. Bassiouny
}

Department of Biochemistry, Medical Research Institute,Alexandria University, 165 El-Horreya Street, Alexandria,

Egypt

Department of Biochemistry, Faculty of science Alexandria University, Bagdad Street Moharam Bek, Alexandria,

Egypt

"Corresponding Author

Rana HM. Khafaga

Department of Biochemistry, Medical Research Institute,

Alexandria University

165 El-Horreya Street, Alexandria,

Egypt

Email: ranahassanmahmoud@gmail.com, ranahassanmahmoud@alexu.edu.eg

Tel: $+201141274363,+201024273634$

Received:18 March 2019; | Revised:05 August 2019; | Accepted:09 March 2020

\begin{abstract}
Pancreatic tumor is a lethal malignancy resistant to conventional current protocols for management including surgical intervention, chemotherapy, and radiation which possesses adverse effects. The present study was designed to evaluate the effects of an extract from Annona Muricata, known as Graviola. Graviola leaves extract (GLE) contain bioactive compound "annonaceous acetogenins" known for anticancer activity on cancer cell lines. This study aimed to explore the molecular mechanisms involved in the progression of pancreatic cancer PC including: redox status, oxidative stress, gene and microRNA expression, and the protective and therapeutic effect of GLE against PC. The study was divided into two phases of male Sprague Dawley rats; to study the protective effect of GLE in one using DMBA for (PC) induction, and GLE effect as adjuvant therapy, blood and pancreatic tissue samples were obtained for assessment of different parameters. These parameters indicated impaired liver and kidney functions in the positive control group (Group II) compared to control (Group I) and co-treated groups (Group III), while in phase II no changes were found, except in treated groups. Also lipid peroxidation, antioxidant capacity, levels of inflammatory cytokines, and expression of NF-kB, COX-2, VEGF-A, iNOS, TNF- $\alpha$ and miRNA levels (miRNA 21, 34a, and 200b) showed aberrant expression in positive control group compared to other groups in both phases. Our findings suggest that microRNAs imbalance in miRNA-21, miRNA-34a and miRNA-200b could be exploited as novel biomarkers for diagnostic and prognostic assessments of PC and as targets for therapy. Collectively, GLE showed significant cytotoxic activity against pancreatic carcinogenesis through multiple pathways motensive nonpregnant women; thereby, exposing them to cardiovascular risks in the near future.
\end{abstract}


Keywords:Pancreatic cancer, 7, 12-Dimethylbenz[a]anthracene, Graviola leaves extract, Orthotopic and Heterotopic implantation

\section{Abbreviations :}

$\begin{array}{llll}\text { PC } & \text { Pancreatic cancer } & \text { TAG } & \text { Triacylglycerol } \\ \text { GLE } & \text { Graviola leaves extract } & \text { EMT } & \text { Epithelial-mesenchymal transition } \\ \text { DMBA } & \text { 7, 12-Dimethylbenz[a]anthracene } & \text { HFD } & \text { High fat diet } \\ \text { NF-KB } & \text { Nuclear factor kappa B } & \text { ROS } & \text { Reactive oxygen species } \\ \text { COX-2 } & \text { Cyclooxygenase-2 } & \text { 3'UTR } & \text { 3' untranslated region } \\ \text { VEGF } & \text { Vascular endothelial growth factor } & \text { H\&E } & \text { Hematoxylin and Eosin stain } \\ \text { iNOS } & \text { Inducible nitric oxide synthase } & \text { PTEN } & \text { Phosphatase and tensin homologue } \\ \text { TNF- } \alpha & \text { Tumor necrosis factor-alpha } & \text { STAT } & \text { signal transducer and activator of transcription } \\ \text { IL-6 } & \text { Interleukin-6 } & \text { MAPK } & \text { Mitogen-activated protein kinase } \\ \text { MiRNA } & \text { micro- RNA } & \text { AKT } & \text { AKT8 virus oncogene cellular homolog } \\ \text { mRNA } & \text { messenger RNA } & \text { PI3-K } & \text { Phosphoinositide 3 kinase } \\ \text { PDAC } & \text { Pancreatic ductal adenocarcinoma } & \text { ZEB-1 } & \text { Zinc finger E-box-binding homeobox 1 } \\ \text { ATP } & \text { Adenosine tri-phosphate } & \text { ZEB-2 } & \text { Zinc finger E-box-binding homeobox 2 } \\ \text { PDAC } & \text { Pancreatic ductal adenocarcinoma } & \text { LDL-C } & \text { Low density lipoprotein } \\ \text { ATP } & \text { Adenosine tri-phosphate } & \text { MDR } & \text { Multi drug resistance } \\ \text { RT-PCR } & \text { Reverse transcriptase } & \text { CFA } & \text { Complete Freund's adjuvant } \\ & \text { polymerase chain reaction } & \text { HDL-C } & \text { High density lipoprotein } \\ \text { ALT } & \text { Alanine transaminase } & \text { TB } & \text { Tumor bearing } \\ \text { AST } & \text { Aspartate transaminase } & \text { PACA-2 } & \text { pancreatic carcinoma-2 } \\ \text { CA-19.9 } & \text { Cancer antigen 19-9 } & \text { CFA } & \text { Complete Freund's adjuvant } \\ \text { ACF } & \text { azoxymethane-induced colonic aberrant } & \text { GSSG } & \text { Oxidized glutathione } \\ & \text { crypt foci } & & \\ \text { GSH } & \text { Reduced glutathione } & & \\ & & & \end{array}$

\section{Introduction}

Pancreatic cancer (PC) is a highly lethal malignancy and it remains the fourth leading cause of cancer-related deaths in the United States and in the world with incidence equaling mortality, as it was estimated, about 53,070 cases of PC were diagnosed and 41,780 deaths occurred in 2016 in the United States ${ }^{[1]}$. Pancreatic cancers are grouped into two main types: exocrine tumors (95\% of all PC) and endocrine tumors, and pancreatic ductal adenocarcinoma (PDAC) were found to be the most common type, making up about $90 \%$ of all exocrine tumors ${ }^{[2]}$. PC is predominantly seen in men and at advanced age and has an aggressive course; it takes the first place among asymptomatic cancers. This type of malignancy is very difficult to be detected as it has no early signs and spreads rapidly to the surrounding organs ${ }^{[3]}$.

The development of pancreatic tumor and progression is multi-factorial because many different pathways are involved but the order and the extent of each pathway are still not clear. Thus, there is an urgent need to understand the molecular mechanisms underlying this disease in order to develop better detection, diagnosis, prognosis as well as therapeutic targets. There are several risk factors found to be associated with the development of PDAC as: obesity, family history, diabetes mellitus, heavy alcohol abuse, smoking, chronic 
pancreatitis and dietary factor, and although many of these risk factors have been identified, the causes of PC are still insufficiently known ${ }^{[4]}$.

Limited evidence-based therapies that are available for the prevention and treatment of PC were found, and in order to develop such therapies, proper animal models are necessary. Increasing clinical efforts have been devoted to improve PC chemotherapy outcomes for over a decade, but regrettably no significant improvements have been reported [5]. It was found that increasing consumption of fruits and vegetables can be associated with a reduced risk of most cancers, including PC. For this reason, the potential effect of natural products in PC therapies has been widely investigated as; graviola leaves extract (GLE) ${ }^{[6]}$.

It has been found that GLE major bioactive components; annonaceous acetogenins, have the ability to manifest efficient and uncommon selective growth inhibition against cancer cells, and also they were found to be toxic to cancer cells by inhibiting the mitochondrial complex I, which is involved in ATP synthesis in these cancer cells. Thus, GLE has been indicated to have promising characteristics for PC therapeutics in combination with chemotherapy ${ }^{[5]}$.

The present study aimed to explore the molecular mechanisms involved in the progression of PC including; disturbed cell signaling, redox status, oxidative stress, inflammatory responses, deregulated gene expression in: Nuclear factor kappa B (NF-K B), Cyclooxygenase-2 (COX-2), Vascular endothelial growth factor (VEGF-A), Inducible nitric oxide synthase (iNOS) and Tumor necrosis factor-alpha (TNF- $\alpha$ ) and disturbed microRNA expression miRNA (miR-21, 34a, and 200b) using orthotropic application of 7, 12Dimethylbenz[a]anthracene (DMBA) in rats to induce pancreatic tumor then the pattern of growth of the subcutaneous tumors was examined using allograft technique (Heterotropic). Also, the study aimed to explore the protective and therapeutic effects of GLE against PC.

\section{Animals and Methods}

\subsection{Chemicals}

DMBA (purchased from: Sigma Company, Germany), Graviola leaves extract (purchased from Bio Nutrition, Inc. Island Park, NY 11558 U.S.A), Gemcitabine chemotherapy (purchased from Actavis Italy S.p.A 20014, Nerviano- Milano, Italy), ALT, AST, total bilirubin, lipid profile, creatinine, lipid peroxidation and total antioxidant capacity (all obtained from Bio Med Diagnostic INC - USA), total protein and albumin (obtained from Spectrum diagnostics), Rat TNF-alpha and IL-6 ELISA kits (obtained from Koma Biotech Inc.), CA19-9 ELISA kit (obtained from Shang Hai Blue Gene Biotech Co.Ltd.), Total RNA extraction (using Biozol total RNA extraction reagent from Bioflux according to the manufacturer instructions), the HiSenScript ${ }^{\mathrm{TM}} \mathrm{RH}(-)$ cDNA Synthesis Kit (obtained from INtRON Biotechnology), primer sets (obtained from Bio Basic Canada Inc.), onestep RT-PCR Master Mix (obtained from GeneDirex), TBE buffer (89.0 $\mathrm{mM}$ Tris-borate EDTA, pH 8.0) from Fermentas Life Science, Agarose (from Molecular Biology Grade); Ethidium bromide (from BioShop Canada Inc. Burlington) DNA ladder (from GeneDirex), miRNA reverse transcription (miScript II RT Kit), miRNA real-time PCR detection (miScript SYBR Green PCR Kit) from Qiagen, and miScript Primer Assay (obtained from Qiagen).

\subsection{Animals}

A total of 72 adult male Sprague-Dawley rats were used (weight ranged from 220-250 grams) and were conducted on 2 phases; phase I: Eighteen rats were used for orthotropic induction of pancreatic tumor using DMBA, and they were divided into 3 groups (6 rats each): Group I: sham control group, Group II: positive control group for PC induction using $3 \mathrm{mg}$ of DMBA, and Group III: co-treated group with GLE using a dose of $200 \mathrm{mg} / \mathrm{kg}$ orally daily for 2 weeks then pancreatic tumor was induced using $3 \mathrm{mg}$ of DMBA, and after induction the same dose of GLE was given orally to each rat daily. Different groups of phase I were fed on 
normal diet with high fat diet (HFD). The HFD contained $20 \mathrm{~g}$ of fat $/ 100 \mathrm{~g}$ of commercial rat chow diet (19 $\mathrm{g}$ of butter and $1 \mathrm{~g}$ of soybean oil to provide essential fatty acids). All components of the high-fat diet were ground and blended), high fat diet was given to promote cancer induction.

In phase II, pancreatic adenocarcinoma obtained from Group II were used for subcutaneous inplantation in new groups of rats. Fifty four rats were divided into 4 groups: Group A: sham control group (6 rats), Group B: positive control group (6 rats) for pancreatic adenocarcinoma tissue induction, Group C: co- treated group (6 rats) that received GLE (200 mg/kg) orally daily for 2 weeks then the subcutaneous implementation with tumor tissues was performed, and after induction, the same dose of GLE was given to each rat daily, and finally Group D: the treated group for pancreatic adenocarcinoma tissue induction. This group was divided into 2 subgroups (18 rats each): Subgroup D (a): to study the effect of Gemcitabine chemotherapy with an intraperitonal dose of 50 $\mathrm{mg} / \mathrm{kg}$, and it was divided into 3 different groups: Group D (Ia): representing treatment after 2 weeks of cancer induction (chemo only $2 \mathrm{~W}$ ), Group D (IIa): representing treatment after 4 weeks of cancer induction (chemo only 4W), Group D (IIIa): representing treatment after 6 weeks of cancer induction (chemo only 6W). Subgroup D (b): to study the effect of Gemcitabine chemotherapy with an intraperitonal dose of $50 \mathrm{mg} / \mathrm{kg}$ with GLE dose $(200 \mathrm{mg} / \mathrm{kg}$ ) orally daily for 2 weeks after cancer induction. This group was divided into 3 different groups: Group D (Ib): representing treatment after 2 weeks of cancer induction (chemo + Graviola 2W), Group D (IIb): representing treatment after 4 weeks of cancer induction (chemo + Graviola 4W), Group D (IIIb): representing treatment after 6 weeks of cancer induction (chemo + Graviola 6W).

At the end of each phase rats were sacrificed to obtain serum samples and pancreatic tissues from different groups and sub-groups. Serum samples were obtained for assessment of ALT, AST, total bilirubin, lipid profile, albumin, total protein, creatinine, total antioxidant capacity, lipid peroxidation, IL-6 protein, TNF-alpha protein, and
CA 19.9 protein. Pancreatic tissues were obtained for RNA extraction, determination of glutathione, and for histopathological investigations (immersed in $10 \%$ neutral formaline solution).

\subsection{Methods}

Biochemical parameters:

Alanine aminotransferase (ALT), aspartate aminotransferase (AST), total bilirubin, lipid profile, creatinine, lipid peroxidation and total antioxidant were determined using reagents obtained from Bio Med Diagnostic INC (USA), according to the manufacturer instructions.

Total protein and albumin were determined using reagents obtained from Spectrum diagnostics, according to the manufacturer instructions.

TNF-alpha and IL-6 were determined using reagents obtained from Koma Biotech Inc. CA19-9 was determined using reagents obtained from Shang Hai Blue Gene Biotech Co.Ltd.), according to the manufacturer instructions.

Total RNA isolation:

Total RNA was isolated from the different tissues using Biozol total RNA extraction reagent from Bioflux according to the manufacturer instructions.

RT-PCR analysis:

For semi-quantitative determination of the gene expression of NF-kB, COX-2, VEGF-A, and TNF- $\alpha$, the one-step RT-PCR Master Mix were used. For NF-KB, the following primer sets were used: forward 5'-CGGCCTCATCCACATGAACT$3^{\prime}$ and reverse 5'-AATGGCTTGCTCCAGGTCTC $-3^{\prime}$. The primer set of VEGF-A are as follows: forward 5' - GTACCTCCACCATGCCAAGT -3' and reverse $5^{\prime}$ - TGTCACATACGCTCCAGGAT - $3^{\prime}$ . The primer set of TNF-alpha are as follows: forward 5'- TCTTCTCATTCCCGCTCGTG $-3^{\prime}$ and reverse 5'- GGTGAGGAGCACATAGTCGG -3'. The primer set of COX-2 are as follows: forward 5'TGATGACTGCCCAACTCCATG -3' and reverse $5^{\prime}$ - AATGTTGAAGGTGTCCGGCA - $3^{\prime}$.To standardize the amount of mRNA in each sample, RT-PCR of glyceraldehyde-3-phosphate 
dehydrogenase (GAPDH) was performed in parallel using the following primer sets: forward $5^{\prime}$ AATGTGTCCG TCGTGGATCTGA $-3{ }^{\prime}$ and reverse 5'- GATGCCTGCTTCACCACCTTCT -3'. At the end of the program, the RT-PCR product was run on $1.5 \%$ agarose and stained with ethidium bromide. The bands were visualized by using a UV plate. The bands were scanned and the data were analyzed using UVP DOC-ITLS TM Image \& acquisition and analysis using gel documentation system software (Ultra-Violet product, Ltd. Cambridge, UK) that analyze the relative band densities of the genes to GAPDH band (as internal control).

Real time PCR:

The relative expression of iNOS was quantified relative to the expression of the reference gene $(\mathrm{GAPDH})$ in the same sample. Primers for iNOS were as follows: forward 5'GCCTCCCTCTGGAAAGA -3' and reverse 5'TCCATGCAGACAACCTT-3'; and for GAPDH, forward 5'- AATGTGTCCGTCGTGGATCTGA -3' and reverse

5 '-

GATGCCTGCTTCACCACCTTCT -3'. Reactions were carried out using SYBR Green PCR Master Mix (Applied Biosystems), $0.5 \mu \mathrm{M}$ of each forward and reverse primer and $50 \mathrm{ng}$ of genomic DNA with the following conditions: $95^{\circ} \mathrm{C}$ for $10 \mathrm{~min}$ followed by 40 cycles of $95{ }^{\circ} \mathrm{C}$ for $15 \mathrm{~min}, 60^{\circ} \mathrm{C}$ for $30 \mathrm{~min}$, and $72{ }^{\circ} \mathrm{C}$ for $30 \mathrm{~min}$. The relative iNOS copy number was calculated using the $\Delta \Delta \mathrm{Ct}$ method, then finally the expression ratio or fold difference in expression was calculated from the formula: Normalized expression ratio $=2^{-\Delta \Delta} \mathrm{Ct}$.

MiRNA analysis:

It enables real-time PCR quantification of mature miRNA (miR 21,34a and 200b) using targetspecific miScript Primer Assays (forward primers) and the miScript SYBR Green PCR Kit, which contains the miScript Universal Primer (reverse primer) and QuantiTect SYBR Green PCR Master
Mix. The miRNA kit (CAT.NO: 217004) was used with miScript PCR Controls. 2x QuantiTect SYBR Green PCR Master Mix, 10x miScript Universal Primer, 10x miScript Primer Assay, template cDNA, and RNase-free water were mixed according to the manufacturer' $\mathrm{s}$ instructions. Reactions were carried out using SYBR Green PCR Master Mix (Applied Biosystems), with the following conditions: $95^{\circ} \mathrm{C}$ for $15 \mathrm{~min}$ followed by 40 cycles of $94{ }^{\circ} \mathrm{C}$ for $15 \mathrm{sec}, 55^{\circ} \mathrm{C}$ for $30 \mathrm{sec}$, and $70{ }^{\circ} \mathrm{C}$ for $30 \mathrm{sec}$ for each cycle. The relative miRNAs copy number was calculated using the $\Delta \Delta$ $\mathrm{Ct}$ method, then finally the expression ratio or fold difference in expression was calculated from the formula: Normalized expression ratio $=2^{-\Delta \Delta \mathrm{Ct}}$.

ELISA measurements:

TNF-alpha and IL-6 were assayed in serum using ELISA kit (Koma Biotech Inc.), while serum CA19-9 was assayed using ELISA kit (Shang Hai Blue Gene Biotech Co.Ltd.) according to the manufacturer's instructions.

Histopathological study:

Pancreatic tissue specimens from each studied group were fixed in $10 \%$ neutral buffered formalin then washed in running water, dehydrated in ascending series of ethyl alcohol, cleared in xylene and embedded in paraffin wax. Sections of $4 \mu \mathrm{m}$ thick were cut using the microtome and stained with Hematoxylin and Eosin stain (H\&E) and Trichrome stain for histopathological investigations.

Statistical Analysis:

The data were expressed as mean \pm SEM. A one-way analysis of variance (ANOVA) with Posthoc test (Bonferroni) was performed on each normally distributed variable to compare the mean values of the different groups. Differences were considered significant at $\mathrm{P}<0.05$. All statistical analyses were performed using SPSS statistical software version 18 (SPSS, Chicago, IL). 


\section{Results}

\subsection{Body weight}

The results of rats' body weight are summarized in Table 1 . The body weight of rats in group II showed a statistically significant rise compared to group I, and group III.

Table 1: Rats' body weight in different groups of phase I

\begin{tabular}{|c|c|c|c|}
\hline Months & Group I & Group II & Group III \\
\hline \hline $\mathbf{1}$ & $246.67 \pm 3.53$ & $243.17 \pm 5.67$ & $227.8 \pm 3.42$ \\
\hline $\mathbf{2}$ & $259.33 \pm 5.42$ & $253.67 \pm 5.42$ & $239.83 \pm 3.46$ \\
\hline $\mathbf{3}$ & $270.67 \pm 3.54$ & $284.33 \pm 5.34^{(\mathrm{a})}$ & $251.17 \pm 3.67^{(\mathrm{b})}$ \\
\hline $\mathbf{4}$ & $282.17 \pm 2.91$ & $325.67 \pm 5.92^{(\mathrm{a})}$ & $262.00 \pm 3.70^{(\mathrm{b})}$ \\
\hline $\mathbf{5}$ & $294.01 \pm 3.09$ & $366.50 \pm 5.72^{(\mathrm{a})}$ & $273.83 \pm 3.91^{(\mathrm{b})}$ \\
\hline $\mathbf{6}$ & $309.17 \pm 3.54$ & $400.83 \pm 3.03^{(\mathrm{a})}$ & $294.50 \pm 2.36^{(\mathrm{b})}$ \\
\hline $\mathbf{7}$ & $329.00 \pm 3.77$ & $452.50 \pm 6.69^{(\mathrm{a})}$ & $328.33 \pm 3.12^{(\mathrm{b})}$ \\
\hline
\end{tabular}

Data were presented as Mean \pm SE Number of rats in each group $=6$

The statistical analysis for the difference between groups using ANOVA test which is significant at $p<0.05$, and highly significant at $p<0.001$

a: Statistically significant difference compared to group I.

b: Statistically significant difference compared to group II

\subsection{Routine parameters}

Phase I:

Alanine aminotransferase (ALT), aspartate aminotransferase (AST), and total bilirubin showed a statistically significant increase in case of group II compared to group I and group III (Table 2). High total protein level indicated certain type of cancer as $\mathrm{PC}$, and the results showed a significant increase in total protein level, and a significant decrease in albumin level in serum of rats in group II, compared to group I, and group III (Table 2). The results revealed a significant increase in the level of serum creatinine in rats of group II compared to group I, and group III (Table 2). Considering lipid profile, the results showed that group II has significantly higher levels of serum triglycerides, total cholesterol and LDL-C compared to group I and group III, on the other hand serum HDL-C showed a significantly lower level in group II, compared to group I and group III (Table 2).
Phase II:

Alanine aminotransferase (ALT), aspartate aminotransferase (AST), and total bilirubin showed a statistically significant increase in case of different treated groups by chemotherapy compared to different treated groups by chemotherapy and GLE, and no significant change was found between group A, group B and group C (Table 2). Total protein level was significantly increased in case of group $\mathrm{B}$ compared to group $\mathrm{A}$, group $\mathrm{C}$ and the different treated groups by chemotherapy and GLE, while serum albumin showed a significant decline in case of different treated groups by chemotherapy compared to different treated groups by chemotherapy and GLE, and no significant change was found between group A, group B and group C (Table 2). Also serum creatinine level was significantly increased in case of different treated groups by chemotherapy compared to different treated groups by chemotherapy and GLE, and no significant change was found between group A, group B and group C (Table 2). 
Table 2: The routine parameters in different groups of phases I and II

\begin{tabular}{|c|c|c|c|c|c|c|c|c|c|c|c|c|}
\hline & & Phase I & & & & & & Phase II & & & & \\
\hline $\begin{array}{c}\text { Parameter } \\
\text { s }\end{array}$ & $\begin{array}{c}\text { Group } \\
\text { I }\end{array}$ & $\begin{array}{c}\text { Group } \\
\text { II }\end{array}$ & $\begin{array}{c}\text { Group } \\
\text { III }\end{array}$ & $\begin{array}{c}\text { Grou } \\
\text { p A }\end{array}$ & $\begin{array}{c}\text { Grou } \\
\text { p B }\end{array}$ & $\begin{array}{c}\text { Gro } \\
\text { up C }\end{array}$ & $\begin{array}{c}\text { Grou } \\
\text { p D } \\
\text { (Ia) } \\
\end{array}$ & $\begin{array}{c}\text { Grou } \\
\text { p D } \\
\text { (Ib) } \\
\end{array}$ & $\begin{array}{c}\text { Grou } \\
\text { p D } \\
\text { (IIa) } \\
\end{array}$ & $\begin{array}{c}\text { Grou } \\
\text { p D } \\
\text { (IIb) } \\
\end{array}$ & $\begin{array}{c}\text { Grou } \\
\text { p D } \\
\text { (IIIa) } \\
\end{array}$ & $\begin{array}{c}\text { Grou } \\
\text { p D } \\
\text { (IIIb) } \\
\end{array}$ \\
\hline ALT(U/L) & $\begin{array}{c}57.6 \pm \\
5.43\end{array}$ & $\begin{array}{c}99 \pm \\
7.02^{\text {(a) }}\end{array}$ & $\begin{array}{c}62 \pm \\
2.45^{(a)}\end{array}$ & $\begin{array}{l}52 \pm \\
3.26\end{array}$ & $\begin{array}{c}64.6 \pm \\
2.2\end{array}$ & $\begin{array}{c}61.5 \pm \\
3.2\end{array}$ & $\begin{array}{l}86 \pm \\
4.37 \\
\left(a^{\prime}\right)\end{array}$ & $\begin{array}{l}59.5 \pm \\
2.4^{\left(b^{\prime}\right)}\end{array}$ & $\begin{array}{c}97.5 \pm \\
6.29 \\
\left(a^{\prime}, c^{\prime}\right)\end{array}$ & $\begin{array}{l}64 \pm \\
4.16 \\
\left(b^{\prime}, d^{\prime}\right)\end{array}$ & $\begin{array}{l}103 \pm \\
2.12 \\
\left(a^{\prime}, c^{\prime}, e^{\prime}\right)\end{array}$ & $\begin{array}{c}68.3 \pm \\
3.22 \\
\left(b^{\prime}, d^{\prime}, f^{\prime}\right)\end{array}$ \\
\hline AST(U/L) & $\begin{array}{c}96.8 \pm \\
4.69\end{array}$ & $\begin{array}{l}231.8 \pm \\
4.69^{\text {(a) }}\end{array}$ & $\begin{array}{l}146.5 \pm \\
6.61^{(a, b)}\end{array}$ & $\begin{array}{c}115.7 \pm \\
4.17\end{array}$ & $\begin{array}{r}128.8 \\
\pm 1.99\end{array}$ & $\begin{array}{c}119.3 \\
\pm \\
4.73\end{array}$ & $\begin{array}{l}185.8 \\
\pm 3.88 \\
\left(a^{\prime}\right)\end{array}$ & $\begin{array}{c}123.3 \pm \\
8.08 \\
\text { (b') }\end{array}$ & $\begin{array}{l}194.3 \\
\pm 7.1 \\
\left(\mathrm{a}^{\prime}, \mathrm{c}^{\prime}\right)\end{array}$ & $\begin{array}{l}125 \pm \\
3.42 \\
\left(b^{\prime}, d^{\prime}\right)\end{array}$ & $\begin{array}{c}195 \pm \\
5.02 \\
\left(a^{\prime}, c^{\prime}, e^{\prime}\right)\end{array}$ & $\begin{array}{c}142.3 \pm \\
4.4 \\
\left(b^{\prime}, d^{\prime}, f^{\prime}\right)\end{array}$ \\
\hline $\begin{array}{c}\text { Total } \\
\text { bilirubin } \\
\text { (mg/dl) }\end{array}$ & $\begin{array}{c}0.46 \pm \\
0.05\end{array}$ & $\begin{array}{l}1.12 \pm \\
0.06^{\text {(a) }}\end{array}$ & $\begin{array}{c}0.54 \pm \\
0.06^{(\mathrm{b})}\end{array}$ & $\begin{array}{c}0.38 \pm \\
0.02\end{array}$ & $\begin{array}{c}0.58 \pm \\
0.04\end{array}$ & $\begin{array}{l}0.5 \pm \\
0.05\end{array}$ & $\begin{array}{c}0.74 \pm \\
0.02 \\
\text { (a') }\end{array}$ & $\begin{array}{c}0.44 \pm \\
0.02 \\
\left(b^{\prime}\right)\end{array}$ & $\begin{array}{c}0.75 \pm \\
0.04 \\
\left(b^{\prime}, d^{\prime}\right)\end{array}$ & $\begin{array}{c}0.47 \pm \\
0.02 \\
\left(b^{\prime}, c^{\prime}, e^{\prime}\right)\end{array}$ & $\begin{array}{c}0.81 \pm \\
0.03 \\
\left(a^{\prime}, c^{\prime}, f^{\prime}\right)\end{array}$ & $\begin{array}{c}0.5 \pm \\
0.06 \\
\left(b^{\prime}, d^{\prime}, f^{\prime}\right)\end{array}$ \\
\hline $\begin{array}{c}\text { Creatinine } \\
\text { (mg/dl) }\end{array}$ & $\begin{array}{c}1.03 \pm \\
0.05\end{array}$ & $\begin{array}{l}2.11 \pm \\
0.11\end{array}$ & $\begin{array}{c}1.4^{ \pm} \\
0.07^{(\mathrm{a}, \mathrm{b})}\end{array}$ & $\begin{array}{c}1.15 \pm \\
0.02\end{array}$ & $\begin{array}{c}1.26 \pm \\
0.05\end{array}$ & $\begin{array}{l}1.2 \pm \\
0.07\end{array}$ & $\begin{array}{c}1.33 \pm \\
0.03^{(\mathrm{a}} \\
{ }^{(2)}\end{array}$ & $\begin{array}{l}1.13 \pm \\
0.02^{\left(\mathrm{b}^{\prime}\right)}\end{array}$ & $\begin{array}{l}1.6 \pm \\
0.04 \\
\left(a^{\prime}, c^{\prime}\right)\end{array}$ & $\begin{array}{l}1.16 \pm \\
0.03 \\
\left(b^{\prime}, d^{\prime}\right)\end{array}$ & $\begin{array}{c}1.66 \pm \\
0.03 \\
\left(a^{\prime}, c^{\prime}, e^{\prime}\right)\end{array}$ & $\begin{array}{c}1.26 \pm \\
0.02 \\
\left(b^{\prime}, d^{\prime}, f^{\prime}\right)\end{array}$ \\
\hline $\begin{array}{c}\text { Total } \\
\text { protein } \\
(\mathrm{g} / \mathrm{dl}) \\
\end{array}$ & $\begin{array}{c}6.99 \pm \\
0.17\end{array}$ & $\begin{array}{c}9.8 \pm \\
0.39^{\text {(a) }}\end{array}$ & $\begin{array}{c}7.98 \pm \\
0.16^{(\mathrm{a}, \mathrm{b})}\end{array}$ & $\begin{array}{c}6.70 \pm \\
0.24\end{array}$ & $\begin{array}{l}8.40 \pm \\
0.21^{(a)}\end{array}$ & $\begin{array}{l}7.04 \pm \\
0.06 \\
\text { (b) }\end{array}$ & $\begin{array}{c}7.50 \pm \\
0.06 \\
(\mathrm{a} ; \mathrm{g})\end{array}$ & $\begin{array}{c}6.10 \pm \\
0.20 \\
\left(\mathrm{~g}^{\prime}, \mathrm{b}\right)\end{array}$ & $\begin{array}{c}8.00 \pm \\
0.04 \\
\left(\mathrm{a}, \mathrm{c}^{\prime}\right)\end{array}$ & $\begin{array}{c}6.90 \pm \\
0.16 \\
\left(g^{\prime}, b^{\prime}, d^{\prime}\right)\end{array}$ & $\begin{array}{c}8.50 \pm \\
0.11 \\
\left(\mathrm{a}^{\prime}, \mathrm{c}^{\prime}, \mathrm{e}^{\prime}\right)\end{array}$ & $\begin{array}{c}7.30 \pm \\
0.20 \\
\left(\mathrm{~g}^{\prime}, \mathrm{b}^{\prime}, \mathrm{d}^{\prime}, \mathrm{f}\right)\end{array}$ \\
\hline $\begin{array}{l}\text { Albumin } \\
\text { (g/dl) }\end{array}$ & $\begin{array}{c}4.82 \pm \\
0.12\end{array}$ & $\begin{array}{l}2.06 \pm \\
0.07^{(\mathrm{a})}\end{array}$ & $\begin{array}{c}3.55^{(\mathrm{a}, \mathrm{b})} \\
0.05^{(\mathrm{a})}\end{array}$ & $\begin{array}{c}4.77 \pm \\
0.04\end{array}$ & $\begin{array}{c}4.24 \pm \\
0.05\end{array}$ & $\begin{array}{c}4.48 \\
\pm \\
0.06\end{array}$ & $\begin{array}{c}3.01 \pm \\
0.06 \\
\left(a^{\prime}, g^{\prime}\right)\end{array}$ & $\begin{array}{l}3.48 \pm \\
0.02 \\
\left(\mathrm{~g}^{\prime}, \mathrm{b}^{\prime}\right)\end{array}$ & $\begin{array}{c}2.60 \pm \\
0.02 \\
\left(\mathrm{a}, \mathrm{c}^{\prime}\right)\end{array}$ & $\begin{array}{c}3.30 \pm \\
0.03 \\
\left(\mathrm{~g}^{\prime}, \mathrm{b}^{\prime}, \mathrm{d}^{\prime}\right)\end{array}$ & $\begin{array}{c}2.70 \pm \\
0.02 \\
\left(\mathrm{a}^{\prime}, \mathrm{c}^{\prime}, \mathrm{e}^{\prime}\right)\end{array}$ & $\begin{array}{c}3.17 \pm \\
0.02 \\
\left(\mathrm{~g}^{\prime}, \mathrm{b}^{\prime}, \mathrm{d}^{\prime}, \mathrm{f}\right)\end{array}$ \\
\hline $\begin{array}{c}\text { Triacylgly } \\
\text { cerol } \\
(\mathrm{mg} / \mathrm{dl}) \\
\end{array}$ & $\begin{array}{c}37.8 \pm \\
1.57\end{array}$ & $\begin{array}{l}248 \pm \\
6.77^{(a)}\end{array}$ & $\begin{array}{c}63.8 \pm \\
2.45^{(\mathrm{a}, \mathrm{b})}\end{array}$ & - & - & - & - & - & - & - & - & - \\
\hline $\begin{array}{c}\text { Cholestero } \\
\mathbf{l} \\
(\mathrm{mg} / \mathrm{dl}) \\
\end{array}$ & $\begin{array}{l}58 \pm \\
1.18\end{array}$ & $\begin{array}{c}162.6 \pm \\
3.1\end{array}$ & $\begin{array}{c}87 \pm \\
2.24^{(\mathrm{a}, \mathrm{b})}\end{array}$ & - & - & - & - & - & - & - & - & - \\
\hline $\begin{array}{l}\text { HDL-C } \\
(\mathrm{mg} / \mathrm{dl}) \\
\end{array}$ & $\begin{array}{c}48.5 \pm \\
1.3 \\
\end{array}$ & $26 \pm \frac{1.5}{\text { (a) }}$ & $\begin{array}{c}41.5 \pm \\
0.76^{(\mathrm{a}, \mathrm{b})} \\
\end{array}$ & - & - & - & - & - & - & - & - & - \\
\hline $\begin{array}{l}\text { LDL-C } \\
\text { (mg/dl) }\end{array}$ & $\begin{array}{c}1.9 \pm \\
0.3\end{array}$ & $\begin{array}{c}87 \pm \\
3.18^{(a)}\end{array}$ & $\begin{array}{l}32.6 \pm \\
2.2^{(\mathrm{a}, \mathrm{b})}\end{array}$ & - & - & - & - & - & - & - & - & - \\
\hline
\end{tabular}

Data were presented as Mean \pm SE Number of rats in each group $=6$

The statistical analysis for the difference between groups using ANOVA test which is significant at $p<0.05$, and highly significant at $p<0.001$

a: Statistically significant difference compared to group I.

b: Statistically significant difference compared to group II.

a': Statistically significant difference compared to group A.

b': Statistically significant difference compared to group D (Ia).

c': Statistically significant difference compared to group D (Ib).

d': Statistically significant difference compared to group D (IIa).

e': Statistically significant difference compared to group D (IIb).

$\mathrm{f}^{\prime}$ : Statistically significant difference compared to group D (IIIa).

g': Statistically significant difference compared to group B 
Table 3: Parameters that include; lipid peroxidation, antioxidant systems, inflammatory cytokines, and tumor marker in different groups of phases I and II

\begin{tabular}{|c|c|c|c|c|c|c|c|c|c|c|c|c|}
\hline \multirow[b]{2}{*}{ Parameters } & \multicolumn{3}{|c|}{ Phase I } & \multicolumn{9}{|c|}{ Phase II } \\
\hline & $\begin{array}{c}\text { Group } \\
\text { I }\end{array}$ & $\begin{array}{c}\text { Group } \\
\text { II }\end{array}$ & $\begin{array}{c}\text { Group } \\
\text { III }\end{array}$ & $\begin{array}{c}\text { Group } \\
\text { A }\end{array}$ & $\begin{array}{c}\text { Group } \\
\text { B }\end{array}$ & $\begin{array}{c}\text { Group } \\
\text { C }\end{array}$ & $\begin{array}{c}\text { Gro } \\
\text { up D } \\
\text { (Ia) }\end{array}$ & $\begin{array}{l}\text { Group } \\
\text { D (Ib) }\end{array}$ & $\begin{array}{c}\text { Grou } \\
\text { p D } \\
\text { (IIa) }\end{array}$ & $\begin{array}{c}\text { Group } \\
\text { D } \\
\text { (IIb) }\end{array}$ & $\begin{array}{c}\text { Group } \\
\text { D } \\
\text { (IIIa) }\end{array}$ & $\begin{array}{c}\text { Group } \\
\text { D (IIIb) }\end{array}$ \\
\hline $\begin{array}{c}\text { Lipid } \\
\text { peroxidation } \\
(\mathbf{n m o l} / \mathbf{m l})\end{array}$ & $\begin{array}{c}3.9 \pm \\
0.3\end{array}$ & $\begin{array}{l}16.5 \pm \\
0.24 \text { (a) }\end{array}$ & $\begin{array}{r}6.2 \pm \\
0.35^{(\mathrm{b})}\end{array}$ & $\begin{array}{l}4.5 \pm \\
0.31\end{array}$ & $\begin{array}{c}15.7 \pm \\
0.38^{\left(\mathrm{a}^{\mathrm{a}}\right)}\end{array}$ & $\begin{array}{c}5.4 \pm \\
0.32 \\
\left(b^{\prime}\right)\end{array}$ & $\begin{array}{c}9.3 \pm \\
0.1 \\
\left(a^{\prime}\right)\end{array}$ & $\begin{array}{c}5.07 \pm \\
0.16 \\
\left(b^{\prime}\right)\end{array}$ & $\begin{array}{c}10.1 \pm \\
0.09 \\
\left(\mathrm{a}^{\prime}\right)\end{array}$ & $\begin{array}{c}5.40 \pm \\
0.30 \\
\left(b^{\prime}, d^{\prime}, f^{\prime}\right)\end{array}$ & $\begin{array}{c}10.4 \pm \\
0.51 \\
\left(a^{\prime}, b^{\prime}, e^{\prime}, g\right)\end{array}$ & $\begin{array}{c}5.8 \pm \\
0.33 \\
\left(b^{\prime}, d^{\prime}, f^{\prime}, h\right)\end{array}$ \\
\hline $\begin{array}{c}\text { Total } \\
\text { antioxidant } \\
(\mathbf{m m o l} / \mathrm{L})\end{array}$ & $\begin{array}{l}6.5 \pm \\
0.028\end{array}$ & $\begin{array}{c}5.9 \pm \\
0.07^{\text {(a) }}\end{array}$ & $\begin{array}{c}6.3 \pm \\
0.056 \\
\text { (b) }\end{array}$ & $\begin{array}{l}6.5 \pm \\
0.05\end{array}$ & $\begin{array}{c}5.9 \pm \\
0.05^{\text {(a) }}\end{array}$ & $\begin{array}{c}6.3 \pm \\
0.03^{(\mathrm{b})}\end{array}$ & $\begin{array}{c}6.17 \\
\pm \\
0.02 \\
\text { (a) }\end{array}$ & $\begin{array}{c}6.4 \pm \\
0.02^{(\mathrm{b})}\end{array}$ & $\begin{array}{c}6.07 \pm \\
0.03 \\
\text { (a) }\end{array}$ & $\begin{array}{c}6.4 \pm \\
0.02 \\
\left(\mathrm{~b}^{\prime}, \mathrm{d}^{\prime}, \mathrm{f}^{\mathrm{p}}\right)\end{array}$ & $\begin{array}{c}5.9 \pm \\
0.04 \\
\left(\mathrm{a}^{\prime}, \mathrm{b}^{\prime}, \mathrm{e}^{\prime}, \mathrm{g}\right)\end{array}$ & $\begin{array}{c}6.4 \pm \\
0.02 \\
\left(b^{\prime}, d^{\prime}, f^{\prime}, h\right)\end{array}$ \\
\hline $\begin{array}{c}\text { Total } \\
\text { glutathione } \\
\text { (nmol/g.tissue) }\end{array}$ & $\begin{array}{l}774.6 \\
\pm 29.3\end{array}$ & $\begin{array}{c}355 \pm \\
13.3^{\text {(a) }}\end{array}$ & $\begin{array}{c}629 \pm \\
14.7 \\
(\mathrm{a}, \mathrm{b})\end{array}$ & $\begin{array}{l}729.8 \\
\pm 19.7\end{array}$ & $\begin{array}{l}347.0^{\left(\mathrm{a}^{\mathrm{*}}\right)} \\
11.9^{(2}\end{array}$ & $\begin{array}{c}638.5 \\
\pm 13.0 \\
\left(b^{\prime}\right)\end{array}$ & $\begin{array}{c}426 \pm \\
11.0 \\
\left(a^{\prime}, c^{\prime}\right)\end{array}$ & $\begin{array}{c}761.4 \\
\pm 26.0 \\
\left(b^{\prime}, d^{\prime}\right)\end{array}$ & $\begin{array}{l}424.7 \\
\pm 10.2 \\
\left(\mathrm{a}^{\prime}, \mathrm{c}^{\prime}, \mathrm{e}^{\prime}\right)\end{array}$ & $\begin{array}{l}662.6 \\
\pm 15.4 \\
\left(b^{b}, d^{\prime}, f^{\prime}\right)\end{array}$ & $\begin{array}{c}335 \pm \\
23.9 \\
\left(a^{\prime}, c^{\prime}, e^{\prime}, g\right)\end{array}$ & $\begin{array}{c}657 \pm \\
22.7 \\
\left(b^{b}, d^{\prime}, f^{\prime}, h\right)\end{array}$ \\
\hline $\begin{array}{c}\text { Oxidized } \\
\text { glutathione } \\
\text { (nmol/g.tissue) }\end{array}$ & $\begin{array}{l}27.4 \pm \\
1.17\end{array}$ & $\begin{array}{l}34.1 \pm \\
3.6^{(\mathrm{a})}\end{array}$ & $\begin{array}{c}29.4 \pm \\
2.3\end{array}$ & $\begin{array}{c}26.5 \pm \\
3.4\end{array}$ & $38 \frac{ \pm 2.5}{\left(\mathrm{a}^{\mathrm{a}}\right)}$ & $\begin{array}{l}30 \pm \\
2.1\end{array}$ & $\begin{array}{l}24.8 \\
\pm 2.2\end{array}$ & $\begin{array}{c}23 \pm \\
0.9^{\left(b^{\prime}\right)}\end{array}$ & $\begin{array}{c}28.6 \pm \\
1.0\end{array}$ & $\begin{array}{c}24 \pm \\
1.0^{\left(\mathrm{b}^{\prime}\right)}\end{array}$ & $\begin{array}{c}33 \pm \\
1.3\end{array}$ & $23 \pm 0.5$ \\
\hline $\begin{array}{c}\text { Reduced } \\
\text { glutathione } \\
\text { (nmol/g.tissue) }\end{array}$ & $\begin{array}{l}720 \pm \\
29.0\end{array}$ & $\begin{array}{c}295 \pm \\
15.9^{\text {(a) }}\end{array}$ & $\begin{array}{c}570 \pm \\
14.3 \\
(\mathrm{a}, \mathrm{b})\end{array}$ & $\begin{array}{l}677 \pm \\
20.4\end{array}$ & $\begin{array}{c}270 \pm \\
13.0^{\left(\mathrm{a}^{\prime}\right)}\end{array}$ & $\begin{array}{c}578 \pm \\
11.4 \\
\left(b^{\prime}\right)\end{array}$ & $\begin{array}{c}373 \\
\pm \\
11 \\
\left(\mathrm{a}^{\prime}, \mathrm{c}^{\prime}\right)\end{array}$ & $\begin{array}{l}710 \pm \\
25.3 \\
\left(b^{\prime}, d^{\prime}\right)\end{array}$ & $\begin{array}{c}367 \pm \\
12.2 \\
\left(a^{\prime}, c^{\prime}, e^{\prime}\right)\end{array}$ & $\begin{array}{c}610 \pm \\
16.7 \\
\left(b^{\prime}, d^{\prime}, f^{\prime}\right)\end{array}$ & $\begin{array}{c}267 \pm \\
24.5 \\
\left(\mathrm{a}^{\prime}, \mathrm{c}^{\prime}, \mathrm{e}^{\prime}, \mathrm{g}\right. \\
,)\end{array}$ & $\begin{array}{c}610 \pm \\
22.8 \\
\left(b^{\prime}, d^{\prime}, f^{\prime}, h^{\prime}\right)\end{array}$ \\
\hline GSH/GSSG & $\begin{array}{c}26.4 \pm \\
1.34\end{array}$ & $\begin{array}{c}9.14 \pm \\
1.10^{(\text {a) }}\end{array}$ & $\begin{array}{l}20 \pm \\
1.57 \\
(\mathrm{a}, \mathrm{b})\end{array}$ & $\begin{array}{c}27.2 \pm \\
2.8\end{array}$ & $\begin{array}{c}7.3 \pm \\
0.77^{\left(a^{\prime}\right)}\end{array}$ & $\begin{array}{l}19.7 \pm \\
1.4^{\left(b^{*}\right)}\end{array}$ & $\begin{array}{c}15.7 \\
\pm 1.4 \\
\left(a^{\prime}, c^{\prime}\right)\end{array}$ & $\begin{array}{c}25.6 \pm \\
1.8 \\
\left(b^{\prime}, d^{\prime}\right)\end{array}$ & $\begin{array}{c}13.4 \pm \\
1.4 \\
\left(a^{\prime}, c^{\prime}, e^{\prime}\right)\end{array}$ & $\begin{array}{c}28.3 \pm \\
3.6 \\
\left(b^{\prime}, d^{\prime}, f^{\prime}\right)\end{array}$ & $\begin{array}{c}7.7 \pm \\
0.9 \\
\left(a^{\prime}, c^{\prime}, e^{\prime}, g\right)\end{array}$ & $\begin{array}{c}26.2 \pm \\
0.8 \\
\left(b^{b}, d^{\prime}, f^{\prime}, h\right)\end{array}$ \\
\hline $\begin{array}{c}\text { TNF-alpha } \\
\text { (pg/ml) }\end{array}$ & $\begin{array}{l}17.4 \pm \\
0.27\end{array}$ & $\begin{array}{l}123 \pm \\
7.1^{(\mathrm{a})}\end{array}$ & $\begin{array}{c}80 \pm \\
2.09 \\
(\mathrm{a}, \mathrm{b})\end{array}$ & $\begin{array}{c}21.4 \pm \\
0.41\end{array}$ & $\begin{array}{l}112.8 \pm \\
7.00^{\left(a^{*}\right)}\end{array}$ & $\begin{array}{c}80.8 \pm \\
1.63 \\
\left(a^{\prime}, b^{\prime}\right)\end{array}$ & $\begin{array}{c}57.9 \\
\pm \\
1.29 \\
\left(\mathrm{a}^{\prime}, \mathrm{b}^{\prime}, \mathrm{c}\right)\end{array}$ & $\begin{array}{c}45 \pm \\
1.85 \\
\left(a^{\prime}, b^{\prime}, c^{\prime}\right)\end{array}$ & $\begin{array}{c}63.7 \pm \\
1.59 \\
\left(a^{\prime}, b^{\prime}, c^{\prime}, e\right. \\
,)\end{array}$ & $\begin{array}{c}45.6 \pm \\
1.23 \\
\left(a^{\prime}, b^{\prime}, c^{\prime}, f^{\prime}\right)\end{array}$ & $\begin{array}{c}65 \pm \\
1.67^{\left(a^{2}\right.}, \\
\left.b^{\prime}, c^{\prime}, e^{\prime}, g^{\prime}\right)\end{array}$ & $\begin{array}{c}62.2 \pm \\
2.97 \\
\left(a^{\prime}, b^{\prime}, c^{\prime}\right)\end{array}$ \\
\hline IL-6 (pg/ml) & $\begin{array}{l}18.4 \pm \\
0.27\end{array}$ & $\begin{array}{c}130 \pm \\
3.91^{\text {(a) }}\end{array}$ & $\begin{array}{c}86.8 \pm \\
1.42 \\
(\mathrm{a}, \mathrm{b})\end{array}$ & $\begin{array}{c}22.5 \pm \\
0.48\end{array}$ & $\begin{array}{c}129 \pm \\
3.47^{\left(a^{\prime}\right)}\end{array}$ & $\begin{array}{c}86.6 \pm \\
1.84 \\
\left(a^{\prime}, b^{\prime}\right)\end{array}$ & $\begin{array}{c}44.3 \\
\pm \\
1.02 \\
\left(a^{\prime}, b^{\prime}, c\right)\end{array}$ & $\begin{array}{c}30 \pm \\
0.98 \\
\left(a^{\prime}, b^{\prime}, c^{\prime}\right)\end{array}$ & $\begin{array}{c}56.3 \pm \\
0.95 \\
\left(\mathrm{a}^{\prime}, \mathrm{b}^{\prime}, \mathrm{c}^{\mathrm{p}}, \mathrm{e}\right. \\
)\end{array}$ & $\begin{array}{c}37 \pm \\
1.3 \\
\left(a^{\prime}, b^{\prime}, c^{\prime}, f^{\prime}\right)\end{array}$ & $\begin{array}{l}59.5 \pm \\
1^{\left(a^{\prime}, b^{\prime}, c^{\prime}\right.}, \\
\left.e^{\prime}, g^{\prime}\right)\end{array}$ & $\begin{array}{c}47 \pm \\
0.91 \\
\left(a^{\prime}, b^{\prime}, c^{\prime}\right)\end{array}$ \\
\hline $\begin{array}{c}\text { CA } 19.9 \\
\text { (U/ml) }\end{array}$ & $\begin{array}{l}3.08 \pm \\
0.17\end{array}$ & $\begin{array}{c}42 \pm \\
2.24^{(\text {(a) }}\end{array}$ & $\begin{array}{c}15.7 \pm \\
2.80^{(\mathrm{a}, \mathrm{b})}\end{array}$ & - & - & - & - & - & - & - & - & - \\
\hline
\end{tabular}

Data were presented as Mean \pm SE Number of rats in each group $=6$

The statistical analysis for the difference between groups using ANOVA test which is significant at $p<0.05$, and highly significant at $p<0.001$

a: Statistically significant difference compared to group I.

b: Statistically significant difference compared to group II.

a': Statistically significant difference compared to group A.

b': Statistically significant different compared to group B.

c': Statistically significant different compared to group C.

d': Statistically significant different compared to group D (Ia).

e': Statistically significant different compared to group D (Ib).

$\mathrm{f}^{\prime}$ : Statistically significant different compared to group D (IIa).

g': Statistically significant different compared to group D (IIb).

h': Statistically significant different compared to group D (IIIa). 
Lipid peroxidation, antioxidant systems, inflammatory cytokines, and CA19-9 tumor marker:

\section{Phase I:}

From this study it was found that the lipid peroxidation parameters showed a significant rise in group II compared to group I and group III, while the antioxidant system showed a significant decline in case of group II compared to group I and group III (Table 3). Glutathione is one of the main antioxidant systems; and in this study it was clear that total, reduced glutathione and GSH/GSSG ratio showed a significant decline in case of group II compared to group I and group III, and a significant increase was found in case of oxidized glutathione in group II compared to group I and group III (Table 3). The level of TNF-alpha, IL-6 and the tumor marker CA19.9 showed a statistically significant increase in case of group II and group III compared to group I. (Table 3).

Phase II:
From the current study it was found that the lipid peroxidation parameter (malondialdehyde), and oxidized glutathione showed a significant rise in group B compared to group A and group C, and the different treated groups by chemotherapy and GLE, while the antioxidant capacity, total glutathione, reduced glutathione and GSH/GSSG ratio showed a significant decrease in case of group $\mathrm{B}$ compared to group $\mathrm{A}$ and group $\mathrm{C}$, and the different treated groups by chemotherapy and GLE (Table 3). TNF-alpha and IL-6 cytokines showed a statistically significant rise in case of group $\mathrm{B}$ and group $\mathrm{C}$ compared to group $\mathrm{A}$ and the different treated groups (Table 3).

\subsection{Tumor size volume}

Table 4 represents the tumor size volume in the different groups of phase II, and it shows a high significant relation was found between group $\mathrm{C}$ and different treated groups compared to group B.

Table 4: Tumor size volume in rats in the different groups of phase II

\begin{tabular}{|c|c|c|c|}
\hline $\begin{array}{c}\text { Groups (Phase II) } \\
\text { After cancer induction }\end{array}$ & $\begin{array}{c}\text { Original tumor } \\
\text { volume }\left(\mathrm{cm}^{3}\right)\end{array}$ & $\begin{array}{c}\text { Tumor volume }\left(\mathrm{cm}^{3}\right) \text { after } 1^{\text {st }} \\
\text { week of tumor induction }\end{array}$ & $\begin{array}{c}\text { Tumor volume }\left(\mathrm{cm}^{3}\right) \text { after } 2^{\text {nd }} \\
\text { week of tumor induction }\end{array}$ \\
\hline Group B & $3.94 \pm 0.009$ & $15.25 \pm 0.3$ & $35.3 \pm 0.48$ \\
\hline Group C & $3.52 \pm 0.008$ & $3.78 \pm 0.01^{(\mathrm{a})}$ & $3.92 \pm 0.01^{\text {(a) }}$ \\
\hline $\begin{array}{c}\text { Groups (Phase II) } \\
\text { After cancer induction } \\
\end{array}$ & $\begin{array}{c}\begin{array}{c}\text { Original tumor } \\
\text { volume }\left(\mathrm{cm}^{3}\right)\end{array} \\
\end{array}$ & $\begin{array}{c}\text { Tumor volume }\left(\mathrm{cm}^{3}\right) \text { after } 1^{\text {st }} \\
\text { week of treatment }\end{array}$ & $\begin{array}{c}\text { Tumor volume }\left(\mathrm{cm}^{3}\right) \text { after } 2^{\text {nd }} \\
\text { week of treatment }\end{array}$ \\
\hline Sub group D (Ia) & $3.56 \pm 0.007$ & $0.88 \pm 0.007^{\text {(a) }}$ & $0.21 \pm 0.005^{\text {(a) }}$ \\
\hline Sub group D (Ib) & $3.50 \pm 0.030$ & $0.57 \pm 0.006^{(\mathrm{a}, \mathrm{b})}$ & $0.02 \pm 0.004^{(\mathrm{a}, \mathrm{b})}$ \\
\hline Sub group D (IIa) & $14.6 \pm 0.200$ & $7.40 \pm 0.030^{(\mathrm{a}, \mathrm{b}, \mathrm{c})}$ & $2.60 \pm 0.030^{(\mathrm{a}, \mathrm{b}, \mathrm{c})}$ \\
\hline Sub group D (IIb) & $15.0 \pm 0.400$ & $4.40 \pm 0.040^{(\mathrm{a}, \mathrm{b}, \mathrm{c}, \mathrm{d})}$ & $0.27 \pm 0.030^{(\mathrm{a}, \mathrm{b}, \mathrm{c}, \mathrm{e})}$ \\
\hline Sub group D (IIIa) & $29.8 \pm 0.100$ & $14.8 \pm 0.100^{(\mathrm{a}, \mathrm{b}, \mathrm{c}, \mathrm{d}, \mathrm{e})}$ & $6.30 \pm 0.040^{(\mathrm{a}, \mathrm{b}, \mathrm{c}, \mathrm{d}, \mathrm{e})}$ \\
\hline Sub group D (IIIb) & $30.0 \pm 0.300$ & $9.20 \pm 0.040^{(\mathrm{a}, \mathrm{b}, \mathrm{c}, \mathrm{d}, \mathrm{e}, \mathrm{f})}$ & $0.56 \pm 0.006^{(\mathrm{a}, \mathrm{b}, \mathrm{c}, \mathrm{d}, \mathrm{e}, \mathrm{f})}$ \\
\hline
\end{tabular}

Data were presented as Mean \pm SE Number of rats in each group $=6$

The statistical analysis for the difference between groups using ANOVA test which is significant at $p<0.05$, and highly significant at $p<0.001$

a: Statistically significant different compared to group B.

b: Statistically significant different compared to group D (Ia).

c: Statistically significant different compared to group D (Ib).

d: Statistically significant different compared to group D (IIa).

e: Statistically significant different compared to group D (IIb).

f: Statistically significant different compared to group D (IIIa). 


\subsection{Molecular parameters}

mRNA gene expression:

In phase I; COX-2 (a), NF-KB (b), VEGF (c), TNF-alpha (c) and iNOS (d) relative gene expression (fold control value) levels in pancreatic tissues showed a statistically significant increase in case of group II and group III compared to group I (Fig. 1).

In phase II; COX-2 (a), NF-KB (b), VEGF (c), TNF-alpha (c) and iNOS (d) relative gene expression (fold control value) levels in pancreatic tissue showed a statistically significant rise in case of group B compared to group A, group C and different treated groups by chemotherapy and GLE (Fig. 3).

miRNA gene expression:

In phase I; the oncogenic microRNA 21 showed a highly significant increase in case of group II and group III compared to group I (Fig. 2a). On the contrary the tumor suppressor miRNA 34a showed a highly significant decrease was found in case of group II and group III compared to group I (Fig. 2b), also the tumor suppressive microRNA$200 \mathrm{~b}$ showed a highly significant decline in case of positive control group II and group III compared to group I (Fig. 2b).

In phase II; miRNA 21 showed a statistically significant rise in case of group B compared to group $\mathrm{A}$, group $\mathrm{C}$ and different treated groups by chemotherapy and GLE (Fig. 4a), while miRNA34a and miRNA-200b showed a statistically significant decline in case of group B compared to group $\mathrm{A}$, group $\mathrm{C}$ and different treated groups by chemotherapy and GLE (Fig. 4b).

\subsection{Histological assessment}

In phase I, histological examination of pancreas in Hematoxylin and Eosin stain (H\&E) stained sections from the group I (Fig. 5a), showed normal histological structure of the pancreatic tissue that was formed of lobules packed with acini and are separated from each other by a delicate connective tissue. The islets of Langerhans appeared as pale stained rounded areas that were surrounded by the acini. The main pancreatic duct was found to be lined by simple columnar epithelium resting on basement membrane and surrounded by connective tissue. In group II (Fig. $5 \mathrm{c}$ ), the stain showed epithelial pancreatic gland with prominent nuclei and vacuolated cytoplasm, pancreatic acinar gland loses its architecture, and new blood vessel showing angiogenesis for cancer growth. In group III (Fig. 5e), the stain showed congested blood vessel with proliferating and inflammatory cells and small necrotic areas.

Sections of the pancreas stained with Masson trichrome from the group I (Fig. 5b) showed delicate collagen fibers in the septa that were found between lobules and in between acini. Collagen fibers were also observed surrounding the blood vessels and in the wall of the main pancreatic duct. There were traces of delicate collagen fibers surrounding the margins of the islets and in between their cells. On the other hand, in group II (Fig. 5d) the stain showed thick dense collagen fibers surrounding the blood vessels and dark blue collagen fibers between undifferentiated exocrine epithelial cells showing the spread of cancer cells all over the gland. In group III (Fig. 5f), the stain showed weak collagen fibers in the septa between lobules, and between acini, with small number of congested blood vessels showing the effect of GLE on cancer cells.

In phase II, as in phase I group A (Fig. 6a), showed normal histological structure of the pancreatic tissue that was formed of lobules packed with acini and separated from each other by a delicate connective tissue. In group B (Fig. 6c), the stain showed epithelial pancreatic gland with prominent nuclei and vacuolated cytoplasm, pancreatic acinar gland loses its architecture, and new blood vessel showing angiogenesis for cancer growth. In group $\mathrm{C}$ (Fig. 6e), the stain showed congested blood vessel with proliferating and inflammatory cells and small necrotic areas.

In different treated group by chemotherapy alone, group D (Ia) (Fig. 7a), the H\&E stain showed some regenerative exocrine gland as the epithelial cell situated in the cell membrane, and dilation between glands was found, also inflammation due to necrosis was found. In group D (IIa) (Fig. 7c), 
the stain showed large area of proliferation of epithelial gland cells, huge number of lymphocytic cells and large area of apoptosis, while in group D (IIIa) (Fig. 7e), the stain showed areas of follicular nodules indicating the progression of cancer cells and large number of inflammatory cells was found due to necrosis. (a)

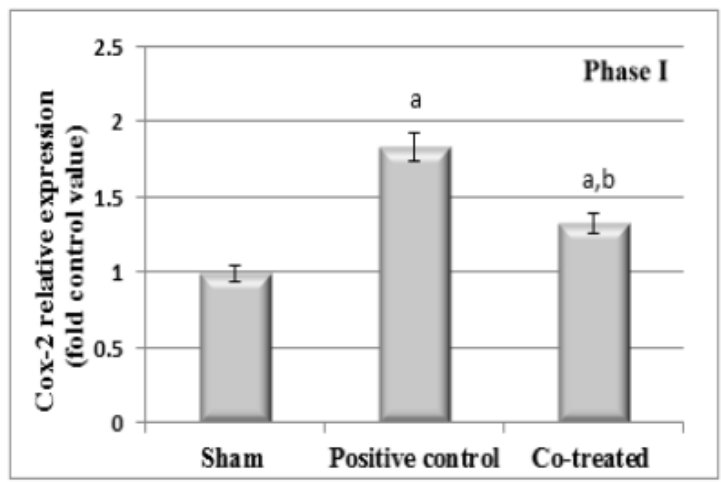

(b)

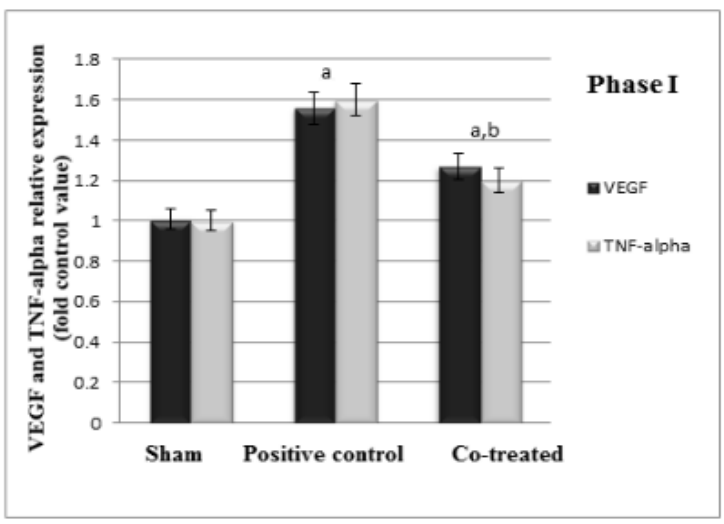

(b)

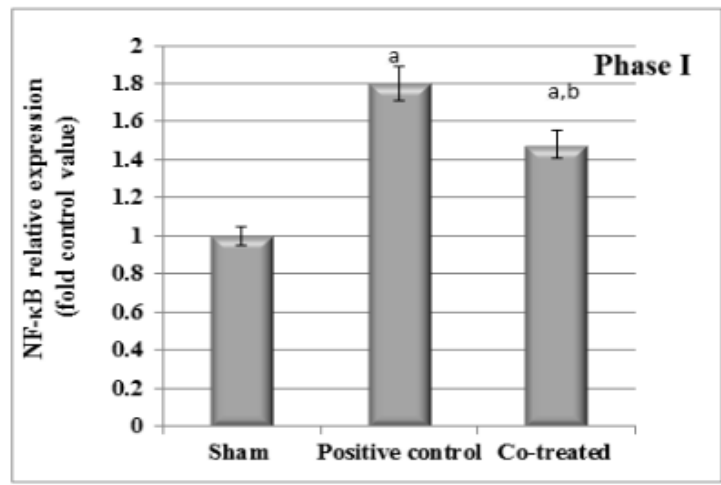

(d)

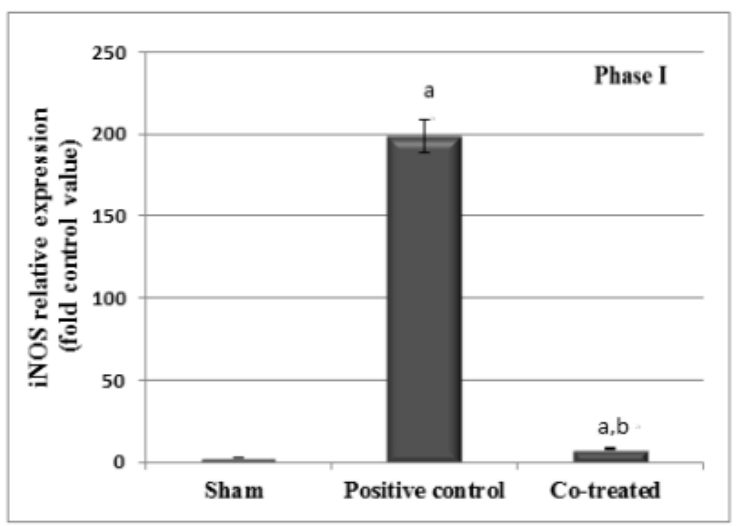

Figure 1: Representing COX-2 (a), NF-KB (b), VEGF (c), TNF-alpha (c) and iNOS (d) relative gene expression (fold control value) in pancreatic tissue in the different groups of phase I. Each bar represents mean of 6 measurements \pm SEM (a and b) represent a significant difference between group II and group III compared to group I, respectively, using ANOVA, $(\mathbf{p}<0.05)$.

a: Statistically significant difference compared to group $I$.

b: Statistically significant difference compared to group II.

In different groups treated by both chemotherapy and GLE, the progression of cancer was found to be with a lesser extent compared to different groups treated by chemotherapy only, in group D (Ib) (Fig. 8a), the stain showed small number of normal features of epithelial cells with dense nuclei indicating regeneration. Areas of apoptotic cells called pykotic cells were also found.
In group D (IIb) (Fig. 8c), the stain showed regeneration of normal pancreatic epithelial cells with large apoptotic areas, while in group D (IIIb) (Fig. 8e), the stain showed loss of pancreatic exocrine gland architecture with large number of inflammatory cells and apoptosis, and large number of blood vessels was found to hemorrhage blood capillaries 
(a)

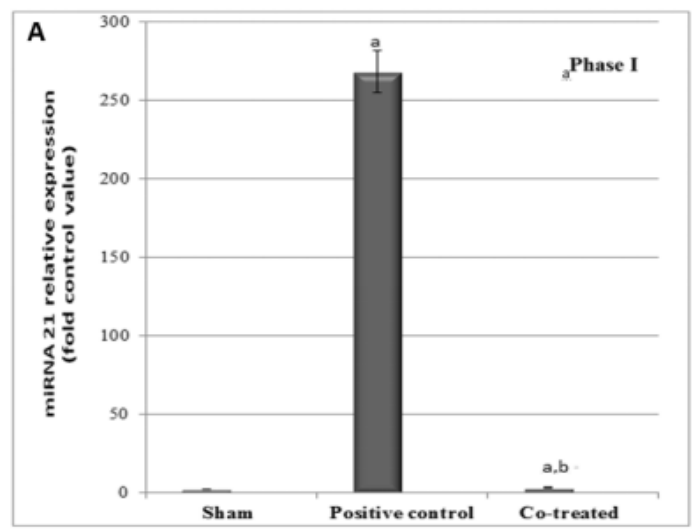

(b)

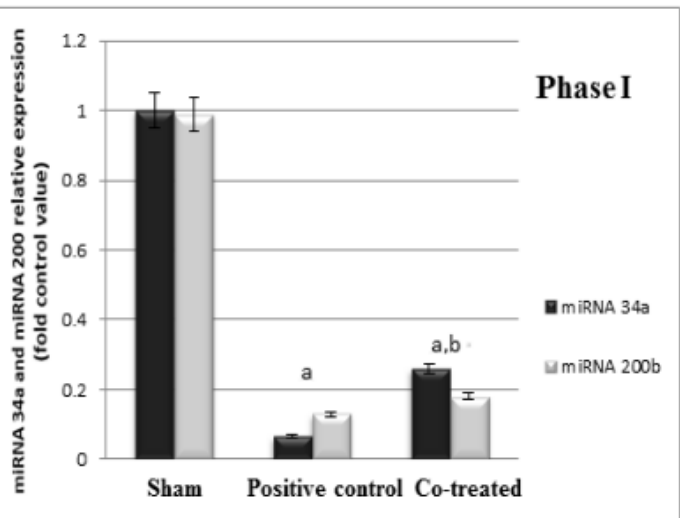

Figure 2: Representing miRNA 21(a), miRNA 34a (b), and miRNA 200b (b) relative gene expression (fold control value) in pancreatic tissue in the different groups of phase I. Each bar represents mean of 6 measurements \pm SEM (a and b) represent a significant difference between group II and group III compared to group I, respectively, using ANOVA, $(\mathbf{p}<0.05)$.

a: Statistically significant difference compared to group $I$.

b: Statistically significant difference compared to group II.

(a)

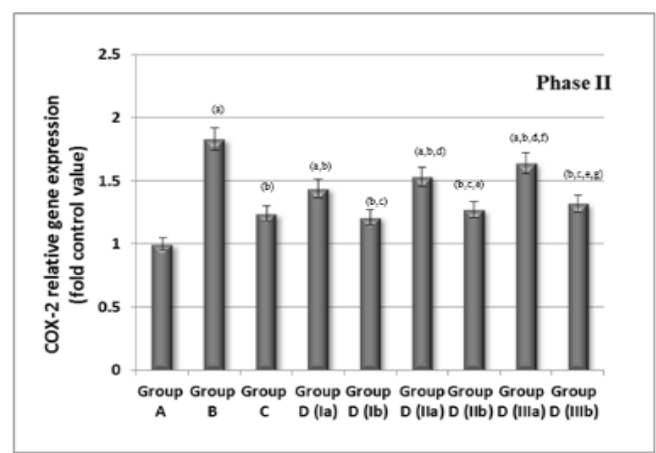

(c)

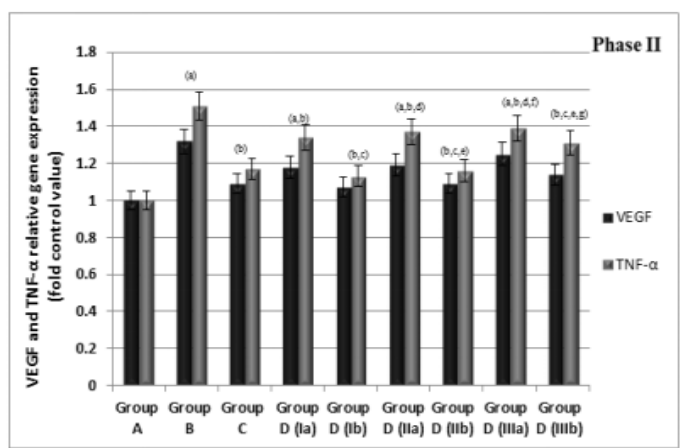

(b)

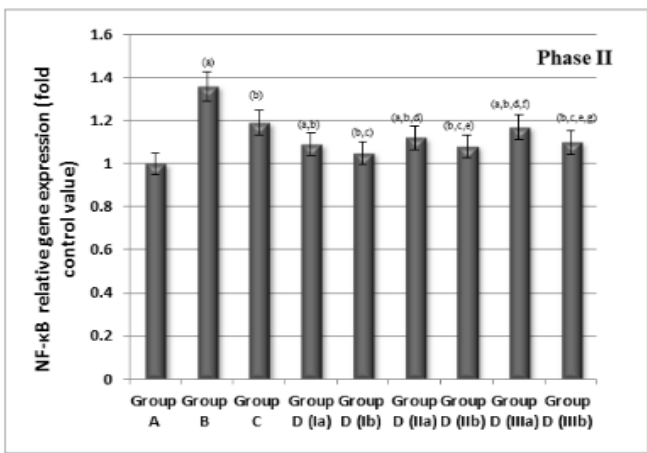

(d)

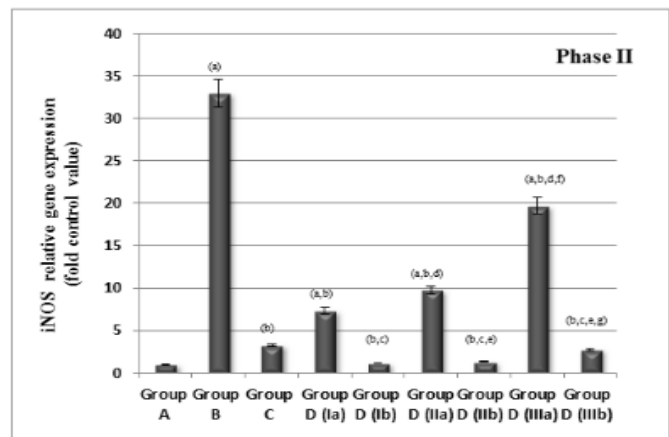

Figure 3: Representing COX-2 (a), NF-кB (b), VEGF (c), TNF-alpha (c) and iNOS (d) relative gene expression (fold control value) in pancreatic tissue in the different groups of phase II. Each bar represents mean of 6 measurements \pm SEM (a, b, c, d, e, f and g) represent a significant difference between different groups as represented, using ANOVA, $(\mathbf{p}<0.05)$.

a: Statistically significant different compared to group A.

b: Statistically significant different compared to group B.

c: Statistically significant different compared to group D (Ia).

d: Statistically significant different compared to group D (Ib). 
e: Statistically significant different compared to group D (IIa).

f: Statistically significant different compared to group D (IIb).

g: Statistically significant different compared to group D (IIIa).

(a)

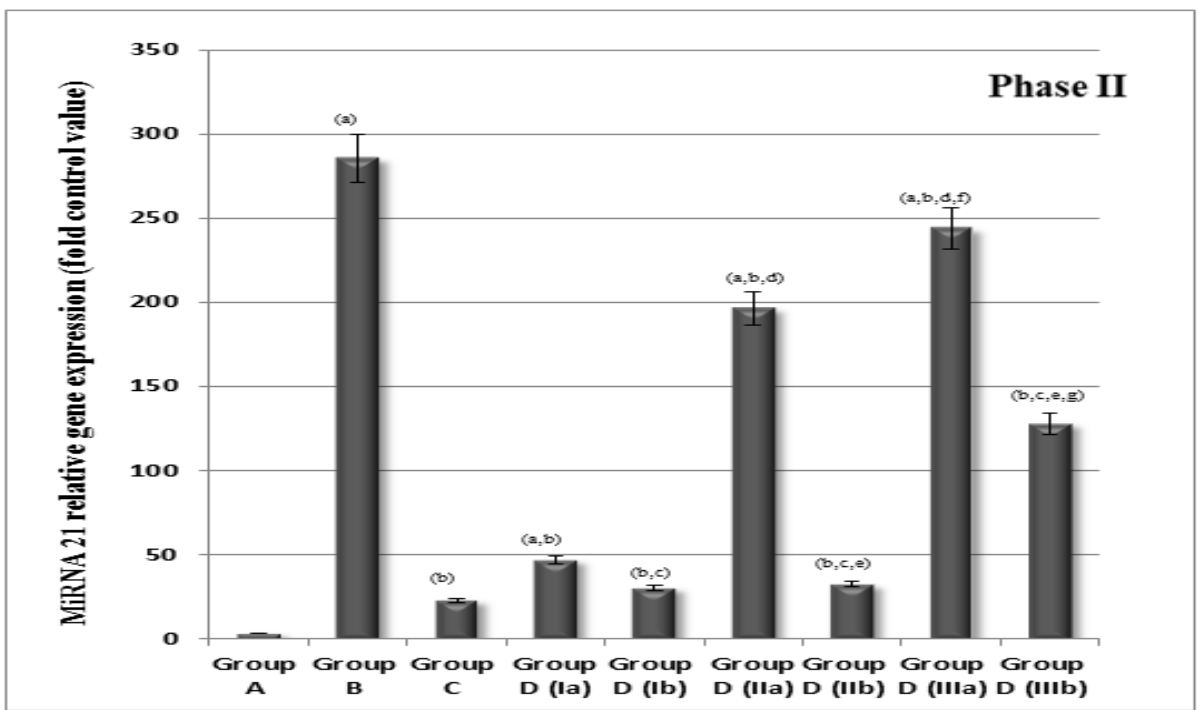

(b)

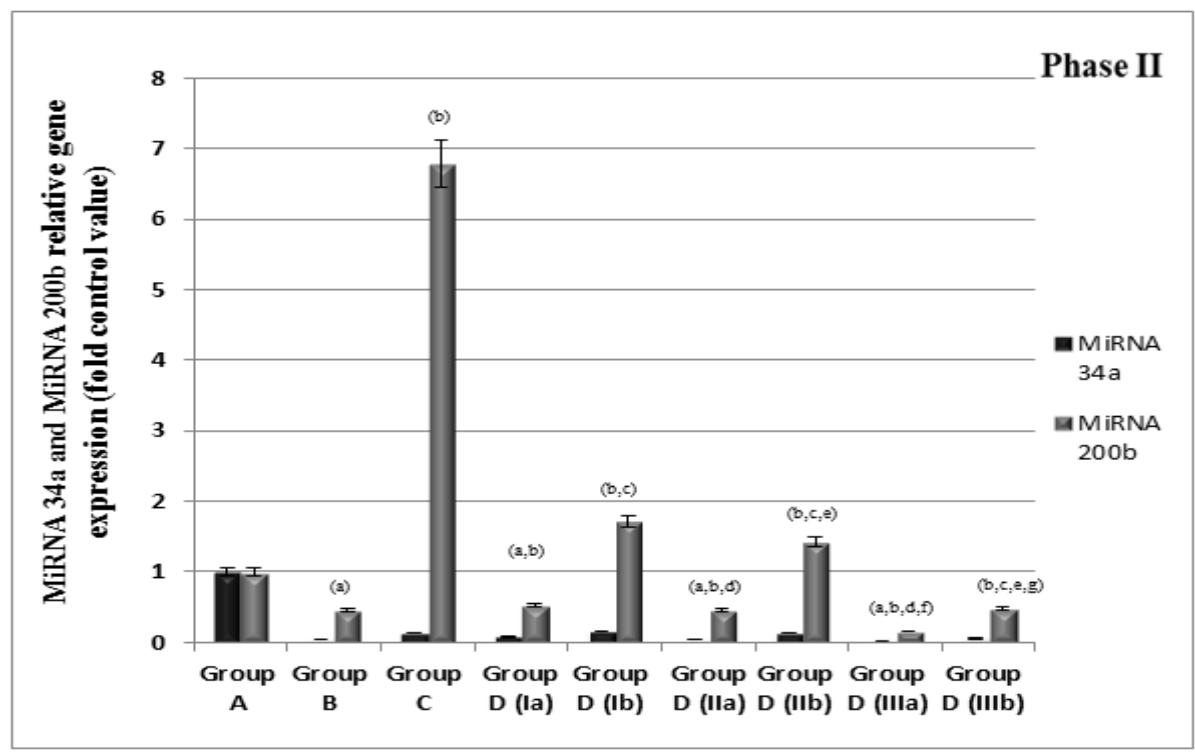

Figure 4: Representing miRNA 21(a), miRNA 34a (b), and miRNA 200b (b) relative gene expression (fold control value) in pancreatic tissue in the different groups of phase II. Each bar represents mean of 6 measurements \pm SEM (a, b, c, d, e, f and g) represent a significant difference between different groups as represented, using ANOVA, $(\mathbf{p}<0.05)$.

a: Statistically significant different compared to group A.

b: Statistically significant different compared to group B.

c: Statistically significant different compared to group D (Ia).

d: Statistically significant different compared to group D (Ib).

e: Statistically significant different compared to group D (IIa).

f: Statistically significant different compared to group D (IIb).

g: Statistically significant different compared to group D (IIIa). 

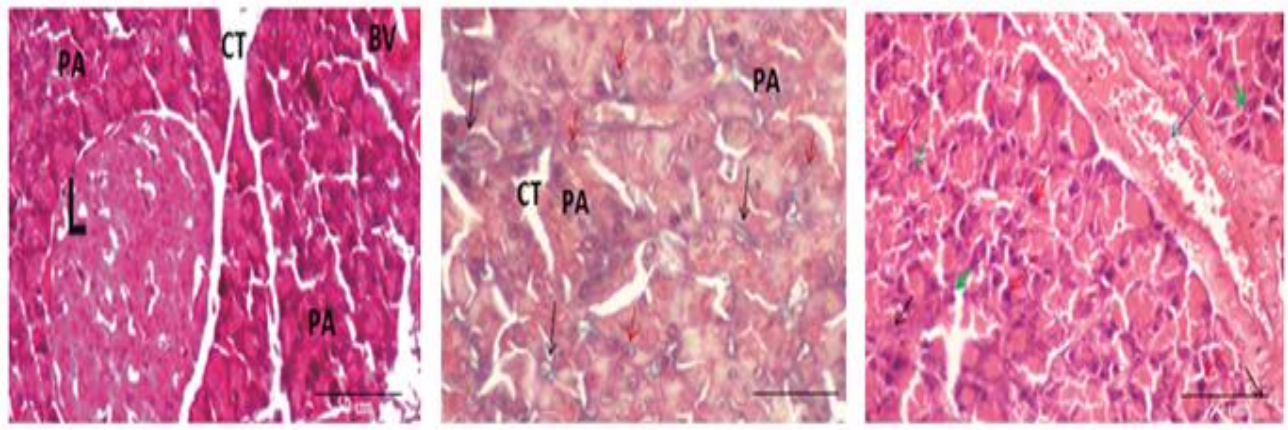

b

d

\section{$f$}
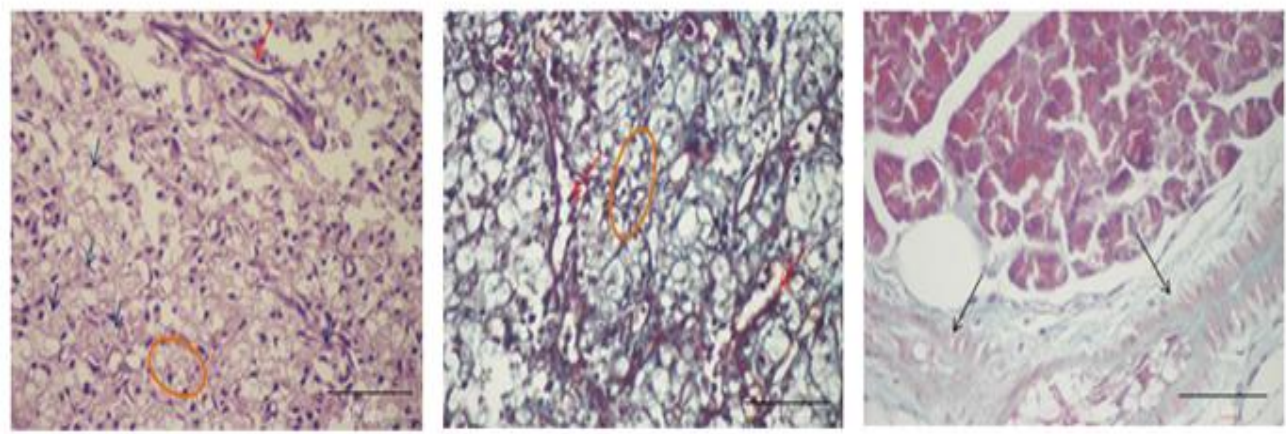

Figure 5: (a) Photomicrographic of a paraffin section of group I showing normal lobular architecture; islets of Langerhans (IS) and the pancreatic acini (PA). Notice the interlobular connective tissue (CT), and blood vessel (BV).(H\&E _ Bar $=50 \mu \mathrm{m})(\mathrm{b})$ Photomicrographic of a paraffin section of group I showing delicate collagen fibers in the septa between lobules, and between acini (black arrows), also showing proliferating cells (red arrows) showing mitosis.(Masson's trichrome _ Bar $=50 \mu \mathrm{m})(\mathrm{c})$ Photomicrographic of a paraffin section of group II showing epithelial pancreatic gland with prominent nuclei and vacuolated cytoplasm, pancreatic acinar gland that loses its architecture (oval shape), new blood vessel showing angiogenesis (red arrow) and many lymphocytic cells (black arrows). $\left(H \& E_{-}\right.$Bar $\left.=50 \mu \mathrm{m}\right)(\mathrm{d})$ Photomicrographic of a paraffin section of group II showing very dense collagen fibers in the septa between lobules, and between acini, with many lymphocytic cells and many blood vessels. Pancreatic gland (oval shape). (Masson's trichrome _ Bar $=50 \mu \mathrm{m})$

(e) Photomicrographic of a paraffin section of group III showing congested blood vessel (blue arrow) with proliferating (green arrows) and inflammatory cells (red arrows), and small necrotic areas (black arrows).(H\&E _ Bar $=50 \mu \mathrm{m})(\mathrm{f})$ Photomicrographic of a paraffin section of group III showing weak collagen fibers in the septa between lobules, and between acini, with congested blood vessels (black arrows).(Masson's trichrome _ Bar $=50 \mu \mathrm{m})$

Also sections stained with Masson trichrome as in phase I; group A (Fig. 6b) revealed delicate collagen fibers in the septa between lobules and in between acini. On the other hand, group B (Fig. 6d) the stain showed thick dense collagen fibers surrounding the blood vessels and dark blue collagen fibers between undifferentiated exocrine epithelial cells showing the spread of cancer cells all over the gland. In group C (Fig. 6f), the stain showed weak collagen fibers in the septa between lobules, and between acini. In different treated group by chemotherapy alone, group D (Ia) (Fig. 7b), the stain showed moderate collagen fibers in the septa between lobules, and between acini with blood vessels, collagen was found to be collected around the area of blood vessel and artery. In group D (IIa) 
(Fig. 7d), the stain showed dense collagen fibers surrounding all lymphocytic cells and between acini. In group D (IIIa) (Fig. 7f), the stain showed thick dense collagen fibers surrounding all lymphocytic cells and between acini. In different groups treated by chemotherapy and Graviola, group D (Ib) (Fig. 8b), the stain showed weak collagen fibers in the septa between lobules, and between pancreatic acini infiltrating it. In group D (IIb) (Fig. 8d)the stain showed weak collagen fibers (blue color) in the septa between lobules, and between acini with small number of blood vessels, while in group D (IIIb) (Fig. 8f), the stain showed moderate collagen fibers in the septa between lobules, and between acini with blood vessels.

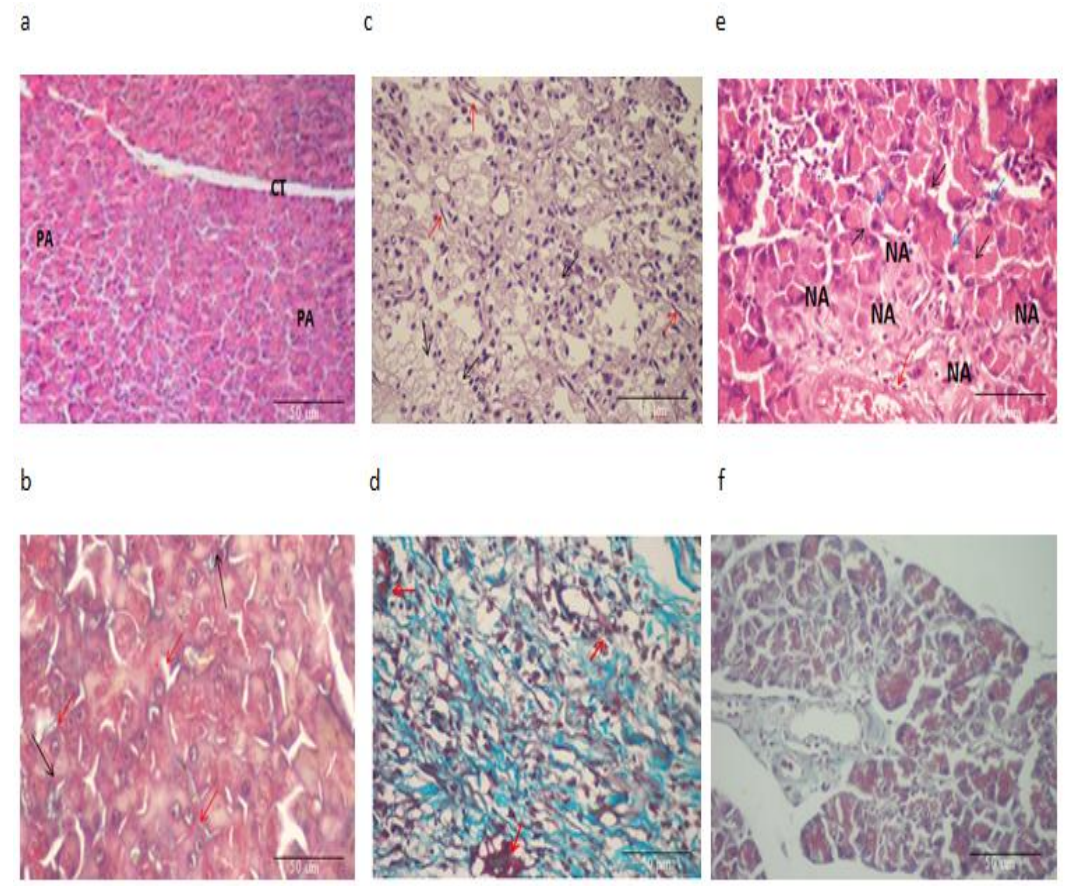

Figure 6: (a) Photomicrographic of a paraffin section of group A showing normal lobular architecture; pancreatic acini (PA). Notice the interlobular connective tissue (CT).(H\&E _ Bar $=50 \mu \mathrm{m})$

(b) Photomicrographic of a paraffin section of group A showing delicate collagen fibers in the septa between lobules, and between acini (black arrows), also showing proliferating cells (red arrows) showing mitosis. (Masson's trichrome proliferated epithelial glandular pancreatic cells with invasive newly formed blood vessels (red arrows), and many lymphocytic cells is present (black arrows).(H\&E _ Bar $=50 \mu \mathrm{m})(\mathrm{d})$ Photomicrographic of a paraffin section of group B showing very dense collagen fibers (blue color) in the septa between lobules, and between acini, with many lymphocytic cells and many blood vessels (red arrows). (Masson's trichrome _ Bar = 50 $\mu \mathrm{m})$ (e) Photomicrographic of a paraffin section of group $C$ showing congested blood vessel (red arrow) with proliferating (black arrows) and some inflammatory cells blue arrows), and necrotic areas (NA). (H\&E _ Bar = $50 \mu \mathrm{m})(f)$ Photomicrographic of a paraffin section of group $\mathrm{C}$ showing weak collagen fibers (faint blue color) in the septa between lobules, and between acini with blood vessels.(Masson's trichrome _ Bar $=50 \mu \mathrm{m})$ 


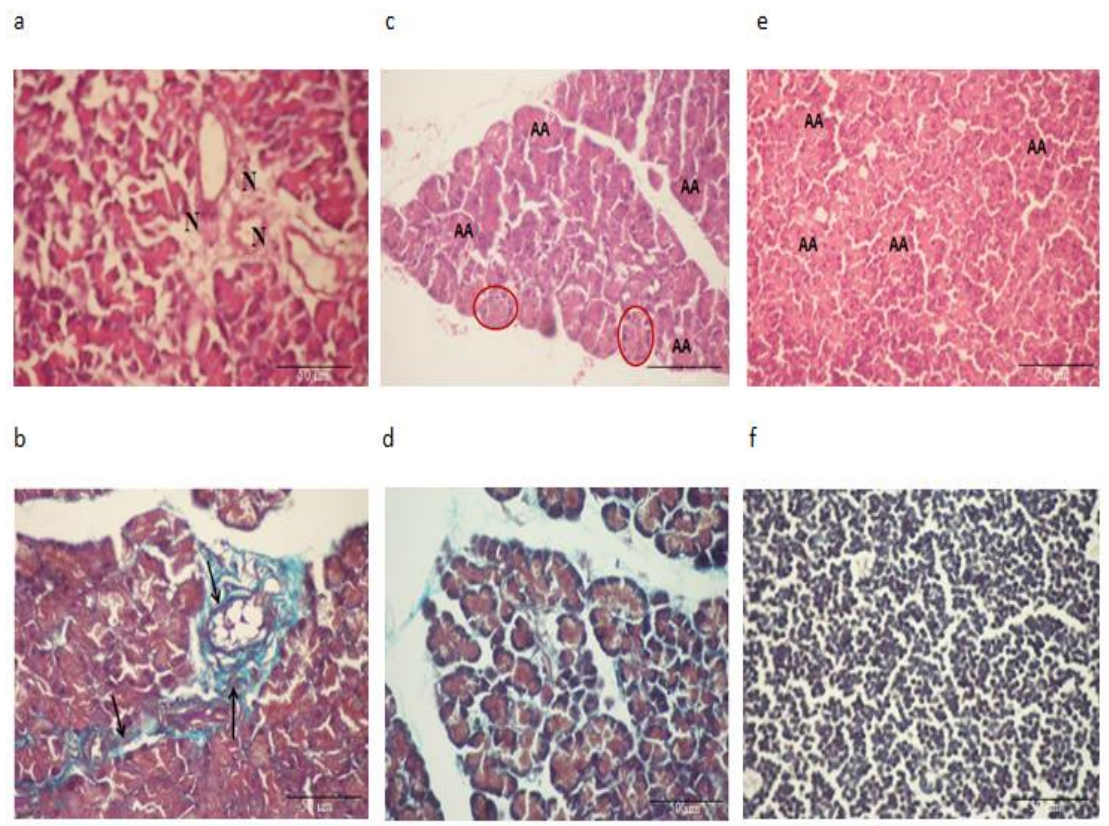

Figure 7: (a) Photomicrographic of a paraffin section of group D (Ia) showing some regenerative exocrine gland as the epithelial cell situated in the cell membrane, and dialation between glands was found. Necrosis is also found $(\mathbf{N})\left(\mathbf{H} \& E_{-}\right.$Bar $\left.=\mathbf{5 0} \mu \mathrm{m}\right)(\mathrm{b})$ Photomicrographic of a paraffin section of group D (Ia) showing moderate collagen fibers (blue color) in the septa between lobules, and between acini with blood vessels. Collagen is collected around the area of blood vessel and artery (black arrows). (Masson's trichrome _ Bar $=\mathbf{5 0} \mu \mathrm{m}$ )

(c) Photomicrographic of a paraffin section of group D (Ib) showing small number of normal features of epithelial cells with dense nuclei indicating regeneration (oval shape). Areas of apoptotic cells are found called pykotic cells $(\mathrm{AA}) .\left(\mathrm{H} \& E_{-}\right.$Bar $\left.=\mathbf{5 0} \mu \mathrm{m}\right)(\mathrm{d})$ Photomicrographic of a paraffin section of group D (Ib) showing weak collagen fibers (blue color) in the septa between lobules, and between pancreatic acini infiltrating it.

(Masson's trichrome _ Bar $=50 \mu \mathrm{m})(\mathrm{e})$ Photomicrographic of a paraffin section of group D (IIa). showing large area of proliferation of epithelial gland cells, huge number of lymphocytic cells and large area of apoptosis (AA).(H\&E _ Bar $=50 \mu \mathrm{m})($ f) Photomicrographic of a paraffin section of group D (IIa) showing dense collagen fibers surrounding all lymphocytic cells (blue color) and between acini. (Masson's trichrome _ Bar $=50 \mu \mathrm{m})$

\section{Discussion}

Pancreatic cancer is a fatal malignancy, and the present work was designed to explore the molecular mechanisms involved in the progression of PC and to evaluate the protective and therapeutic effects of GLE in male Sprague Dawley rats using orthotropic application of the carcinogen DMBA to induce pancreatic tumor then the pattern of growth of the subcutaneous tumors was examined using allograft technique.

The carcinogenic DMBA was found to induce cancer by covalent binding to DNA forming DNA adducts, these adducts lead to mutations in oncogenes and tumor suppressor genes ${ }^{[7]}$.
Interactions of DMBA with DNA can be altered by dietary and environmental factors. A HFD was found to have a promoting effect on tumor development in $\mathrm{PC}{ }^{[8,9]}$. As in rats, diet has been found to play an important factor in hamster PC model; HFD causes enhanced tumor formation which promotes pancreatic carcinogenesis ${ }^{[10]}$.

The present data are in accordance with previous studies which found that the direct implantation of the DMBA into the head of the pancreas causes tubular complexes in acini and induces pancreatic neoplasms of ductal phenotype in which cytokeratin 19 is expressed ${ }^{[11]}$ and K-ras gene mutations are present ${ }^{[8]}$. 


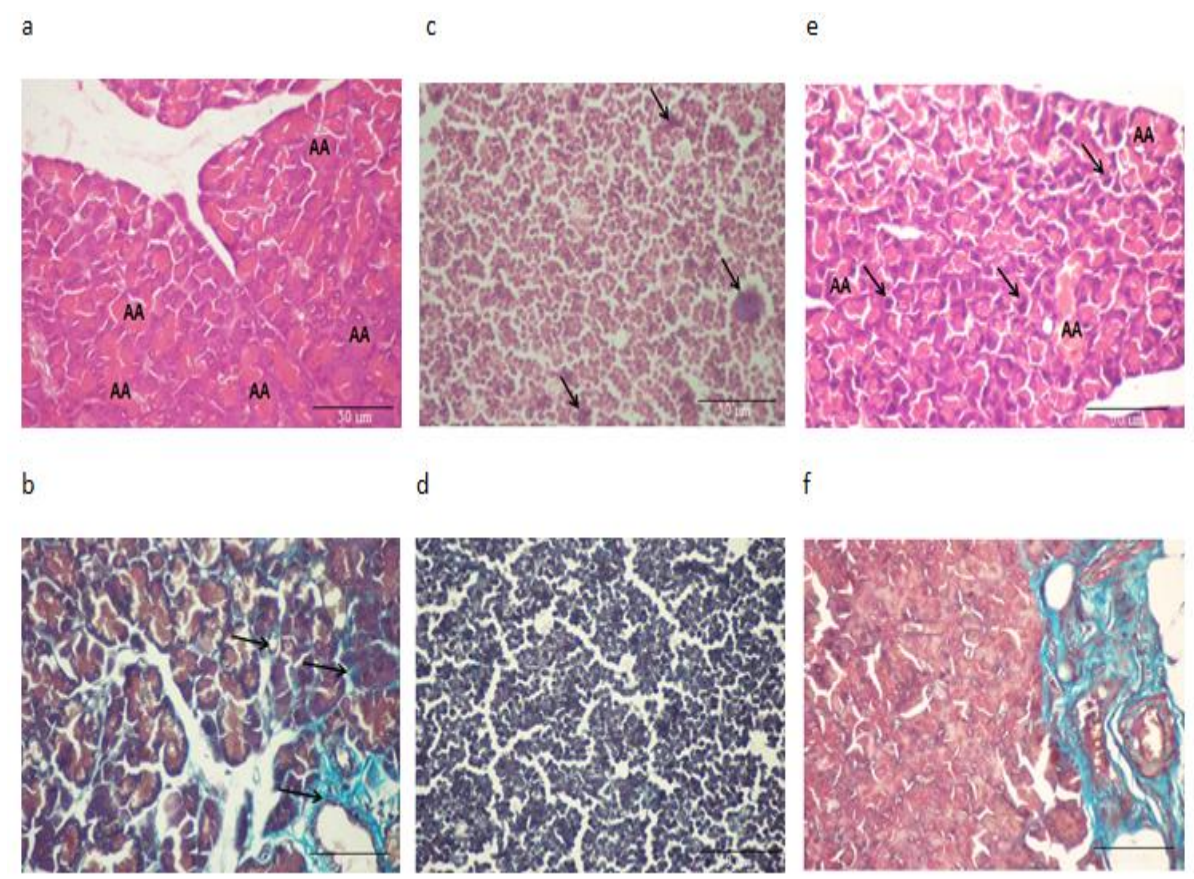

Figure 8: (a) Photomicrographic of a paraffin section of group D (IIb) showing regeneration of normal pancreatic epithelial cells with large apoptotic areas (AA).(H\&E_ Bar $=50 \mu \mathrm{m})(\mathrm{b})$ Photomicrographic of a paraffin section of group D (IIb) showing weak collagen fibers (blue color) in the septa between lobules, and between acini (black arrows) with small number of blood vessels.(Masson's trichrome _ Bar $=\mathbf{5 0} \mu \mathrm{m}$ )

(c) Photomicrographic of a paraffin section of group D (IIIa) showing areas of follicular nodules (black arrows) and large number of inflammatory and lymphocytic cells. $(H \& E \ldots$ Bar $=50 \mu \mathrm{m})(\mathrm{d})$ Photomicrographic of a paraffin section of group D (IIIa) showing very dense collagen fibers surrounding all lymphocytic cells (blue color) and between acini.(Masson's trichrome _ Bar $=50 \mu \mathrm{m})(\mathrm{e})$ Photomicrographic of a paraffin section of group D (IIIb) showing loss of pancreatic exocrine gland architecture with large number of inflammatory cells (black arrows) and apoptosis (AA), and large number of blood vessels found that hemorrhage blood capillaries. $(H \& E$ _ Bar $=50 \mu \mathrm{m})(\mathrm{f})$ Photomicrographic of a paraffin section of treated group D (IIIb) showing moderate collagen fibers (blue color) in the septa between lobules, and between acini with blood vessels.(Masson's trichrome _ Bar $=50 \mu \mathrm{m})$

In the DMBA treated rats of phase I, the results demonstrated that liver function tests; ALT, AST and bilirubin were severely elevated while serum albumin level was declined which indicate liver damage. Also serum creatinine level was significantly elevated as an indication of kidney damage. Serum total protein showed increased level, while abnormal serum lipid profile as indicated by significantly higher levels of triacylglycerol (TAG), total cholesterol and lowdensity lipoprotein cholesterol (LDL-C) and by lower level of high -density lipoprotein-cholesterol (HDLC). These derangements in lipid profile ultimately resulted from the HFD used in the study to promote the action of DMBA.

The orthotropic induction of pancreatic tumor using DMBA results in shifting in the redox status of the pancreatic tissues into more oxidizing environment and up-regulation of TNF- $\alpha$ and IL-6 levels in circulation. The results showed enhanced expression of NF- $\mathrm{KB}$ and its downstream targets COX-2, VEGF-A, iNOS and TNF- $\alpha$. At the level of micro-RNAs, DMBA-induced pancreatic cancer model in rats used in the present study causes significant up-regulation of the oncogenic miRNA; miRNA-21 and significant suppression of tumor suppressor miRNA-34a and miRNA-200b.

The abnormal liver functions may result from the diffusion of DMBA to reach portal circulation and hepatocytes that induce liver dysfunction or may be secondary to the induction of PC. It was found that there is a very close and complex relationship between liver and pancreas, that's why 
the liver function of most of the patients with pancreatic carcinoma was abnormal. Previous studies showed that pancreatic carcinoma can cause acute pancreatitis, in which apoptosis of the pancreatic acinar cells releases trypsin, further promoting the release of a large number of inflammatory cytokines that cause liver injury ${ }^{[11,12,13]}$.

The depletion of reduced glutathione (GSH) could result from: depressed de novo synthesis due to either reduced availability of substrates, or inhibition in the key enzymes, increase the conjugation of GSH with electrophilic toxicants and removal from the body, and (or) due to increased detoxification of free radical and its oxidation into oxidized glutathione $(\mathrm{GSSG})^{[14]}$.

The depletion of GSH system in the present study was associated with significant elevation of lipid peroxidation which indicated a state of oxidative stress. The malignant transformation of pancreatic tissues was associated with decline of the circulatory total antioxidant capacity which indicated an overall oxidative stress on the body.

TNF- $\alpha$ has been found to be the key mediator of inflammation and cancer ${ }^{[15]}$. Constitutive production of TNF- $\alpha$ from the tumor microenvironment is a characteristic of many malignant tumors and its presence is often associated with poor prognosis. TNF- $\alpha$ receptors are expressed on both epithelial and stromal cells. Tumor stromal and epithelial cells, including macrophages generate several inflammatory cytokines such as TNF- $\alpha$ and IL-6, and they recruit more inflammatory cells to the tumor microenvironment to further enhance the proliferation and survival of tumor cells ${ }^{[15]}$.

Nuclear factor-kappa B signaling pathway is one of the pathways that is activated by TNF- $\alpha$. The data of the present study clearly indicated that the upregulation of circulatory TNF- $\alpha$ is associated with enhanced gene expression of NF- K B and its downstream targets genes in the pancreatic tumor tissues as a result of orthotropic application of DMBA. NF- K B was found to be constitutively activated in most human pancreatic cancer tissues but not in normal pancreatic tissues and cells. It is a critical transcription factor that senses any redox imbalance due to carcinogen and facilitates cytokine gene induction during cellular stress, that's why it is called the real culprit of the cell. Oxidative stress activates NF-KB, which regulates the expression of
iNOS, COX-2, VEGF-A, IL-6 and TNF- $\alpha$ by binding to their promoter region ${ }^{[16]}$. The present data indicated significant elevation in the gene expression of these genes as a result of orthotropic application of DMBA on pancreatic tissues.

Interleukin-6, which is the major effector molecule of NF-KB, itself causes NF-KB activation in cancer cells, which results in more IL-6 production as well as more induction of $\mathrm{COX}-2$ and VEGF-A for angiogenesis ${ }^{[17,18]}$. IL-6 is secreted by $\mathrm{T}$ cells and macrophages to stimulate immune response. The primary sources of IL-6 are tumor cells as well as tumor-associated macrophages ${ }^{[19]}$. IL-6 induces its effects through binding to its receptor on the plasma membrane and activating the associated Jaks, which phosphorylate themselves and the receptor that activate signal transducer and activator of transcription (STAT) phosphorylation by Mitogen-activated protein kinase (MAPK) and PI3K/Akt. Phosphorylated STATs dimerize and translocate into the nucleus to regulate target gene transcription ${ }^{[20,21]}$.Elevated levels of IL-6 in serum are found to be associated with epithelial-tomesenchymal transition (EMT) and invasion along with increased size of tumors, metastasis and decreased survival ${ }^{[22]}$.

One of the main target genes that is activated by STATs and NF-KB is COX-2 gene ${ }^{[23,24]}$. The results showed enhanced expression of COX-2 in pancreatic tumors induced by orthotropic application of DMBA. These results are in line with the previous data that indicated up-regulation of COX-2 gene expression in human pancreatic tumors compared with barely detectable levels in normal pancreatic tissue ${ }^{[25]}$. It was found that the expression of phospholipase A2, was higher in pancreatic ductal adenocarcinomas compared with normal pancreatic tissue ${ }^{[26]}$.

The results of the present study also indicated up-regulation of VEGF gene expression in pancreatic tumor tissue. STAT-3 activation by IL-6 facilitates angiogenesis in $\mathrm{PC}$ by inducing the expressions of VEGF-A in tumor-associated endothelial cells ${ }^{[17,27]}$, NF- $\mathrm{KB}$ also controls the expression of VEGF-A which is also involved in cell survival, apoptosis, invasion, metastasis, and angiogenesis ${ }^{[24]}$. VEGF-A is responsible for angioblast differentiation, it is overexpressed in exocrine pancreatic tumors, and due to its high 
sensitivity and specificity it was found to a good biomarker for early detection, prevention, and cure for neoplasms of the pancreas ${ }^{[28]}$. Therefore; NF-KB and IL-6 appear to be cooperated in the up-regulation of VEGF-A ${ }^{[29]}$.

Inducible nitric oxide synthase is another target of NF-KB, which is evidenced in the present study to be highly expressed in pancreatic tumor after exposure to DMBA. iNOS expression is known to be regulated at the expressional level by hypoxia, oxidative stress and inflammatory cytokines (TNF- $\alpha$ and IL-6) ${ }^{[24]}$. NO at high concentrations is believed to modulate immune-mediated anti-tumor activities and angiogenesis [30,31]. Post-transcriptional regulation of iNOS gene expression occurs via mechanisms that influence iNOS mRNA stability $[32,33]$.

The present data was found to be in accordance with others which indicated that the overexpression of miRNA-21 was identified in most types of human carcinomas $^{[34]}$, and NF- $\mathrm{KB}$ activation is also reported in all of these cancers ${ }^{[35]}$, indicating the interplay of miRNA-21 and NF-KB in cancer. MiRNA-21 downregulate PTEN expression that cause activation of AKT and MAP kinase pathway resulting in increased NF-kB activity ${ }^{[36]}$. MiRNA-21 was documented to be one of the top aberrantly expressed miRNAs that displayed increased expression in pancreatic tumors which localized to tumor cells, and it is associated with poor survival of pancreatic adenocarcinoma patients ${ }^{[37]}$.

On the contrary to miRNA-21, miRNA-34a was found to be severely declined in PC tissues in the present model. It is a significant component of the p53 transcriptional network and during DNA damage, and it is commonly deleted in human cancers such as PC ${ }^{[38]}$. MiRNA-34a responsive genes are highly enriched for those that regulate cell-cycle progression, apoptosis, DNA repair, and angiogenesis [39]. Interestingly, miRNA-34a restoration led to an $87 \%$ reduction of the tumor population, and since more than $50 \%$ of primary human cancers have mutations inactivating p53 function, these findings provided impetus to explore the functional restoration of miR-34 as a novel approach to inhibit cancers with p53 loss of function ${ }^{[40]}$.

Histological analysis in phase I revealed typical features of pancreatic malignant transformation as the lesions are characterized by epithelial pancreatic gland with prominent nuclei and vacuolated cytoplasm, acinar gland loses its architecture, new blood vessel showing angiogenesis for cancer growth. The lesions resemble carcinoma at the cytonuclear level, but invasion through the basement membrane is absent.

The histological data were confirmed by the results of cancer antigen 19.9 (CA19.9) which showed significant elevation in the DMBA-treated rats. CA19.9 is expressed in small amounts on the surface of normal epithelial cells, but with malignant transformation, large amounts of CA19.9 are produced at these sites, which results in increased serum levels ${ }^{[41]}$.

The results of phase II study showed that, tumor bearing rats have normal liver and kidney functions while the tumors were growing in size and histologically resemble that of the original pancreatic tumor. Also, the tumor bearing rats exhibited a highly significant elevation in the levels of TNF- $\alpha$ and IL-6. The redox status of allografted tumor tissues showed the same shift to oxidizing environment as that observed in the original which indicated by a significant decrease in the levels of total and reduced GSH and the redox ratio (GSH/GSSG), while a significant increase in the level of GSSG. At the molecular level, the tumor tissues obtained from tumor bearing rats showed similar epigenetic changes in the gene expression of NF-kB, VEGF-A, COX2, iNOS and TNF- $\alpha$ which showed up-regulation to a similar extent observed in phase I tumors. Also, the miRNAs showed similar pattern of change that observed in phase I; the allografted tumor tissues retained the enhanced expression of miRNA-21, miRNA-301a and miRNA-486-5p, while a suppressed expression of miRNA-34a and miRNA-200b.

At the histological level, the administration of GLE during DMBA-induction of PC in phase I resulted in lower inflammatory and milder carcinogenesis with small necrotic area observed in the tumor tissues, also showed weak collagen fibers in the septa between lobules and between acini with small number of congested blood vessels. Similar results were also observed in phase II study which confirmed the protective effects of GLE against the development of heterotropic allograft of pancreatic tumor subcutaneously. The therapeutic effects were also evidenced through co-administration with 
Gemicitabine chemotherapy. The results indicated that, GLE adjuvant treatment enhance the efficiency of chemotherapy in eradicating cancer tissues as evidenced by lower tumor volume in co-GLE treated rats compared to chemotherapy treated rats. The results also clearly demonstrated the superior effects of GLE treatment in the early stage tumor ( 2 and 4 weeks) compared to the late stage (6 weeks) as evidenced by tumor volume and histological studies.

GLE application during phase I significantly ameliorate the deregulated lipid profile, hepatotoxicity and nephrotoxicity associated with DMBA-induced pancreatic carcinogenesis. Also, in phase II the adjuvant use of GLE significantly protect against the toxic effects of chemotherapy on remote organs such as liver and kidney.

It was documented that, annonaceous acetogenins especially annonacin, is effective in killing tumors that have proven resistant to anticancer agents, and have a special affinity for such resistant cells . Actually cancer cells that survive chemotherapy develop resistance to chemotherapy used, called multi-drug resistance (MDR). MDR developed by cancer cells is developed by creating an intercellular pump that pushes anticancer agents out of the cell before chemotherapy can kill it. Although only $2 \%$ of the cancer cells might develop this pump, but these $2 \%$ can eventually expand and grow to create multi-drug-resistant tumors ${ }^{[42]}$. Some of these acetogenins are capable of shutting down these intercellular pumps, thereby killing multidrug resistant tumors; by inhibiting the mitochondrial complex I, which is involved in ATP synthesis so blocking the transfer of ATP into cancer cells, while on the other hand normal cells seldom develop such a pump; therefore, they don't require large amounts of energy to develop this pump ${ }^{[42]}$.

All of the above mentioned data confirm the anti-inflammatory and anti-oxidant effects of GLE. These results are in accordance with reports that support the anti-cancer effects of Graviola extracts [43-45]. The protective effect of the GLE against Complete Freund's adjuvant (CFA)-induced arthritis in rats and xylene-induced ear edema in mice was associated with an attenuation in the TNF- $\alpha$ and IL-1 $\beta$ protein expression, demonstrating that the leaves could be used against both acute and chronic inflammation $^{[43]}$.

It was revealed that, muricoveacin and murihexocin $\mathrm{C}$ isolated from the leaves for Annona muricata displayed cytotoxicity against pancreatic carcinoma (PACA-2) ${ }^{[44]}$. Another study documented the antitumor effect of GLE against chemical carcinogenesis induced by DMBA. The protective effect against DNA damage induced by DMBA showed that oral administration of the GLE may have protective effects towards the development of breast carcinogenesis ${ }^{[44]}$. The leaves, even at the low dose of $30 \mathrm{mg} / \mathrm{kg}$ suppressed the initiation and promotion stage of skin papillomagenesis in mice. The oral administration of the extract at two doses $(250$ and $500 \mathrm{mg} / \mathrm{kg}$ ) for 60 days significantly reduced azoxymethaneinduced colonic aberrant crypt foci (ACF) formation in rats, as assessed by methylene blue staining of colorectal specimens ${ }^{[45]}$.

\section{Conclusion}

Form this study it became clear that, the pancreatic carcinogenesis using orthotropic DMBA application followed by heterotopic allograft is a representative model of human PC and is associated with severe derangements in the cellular signaling pathways including miRNAs levels. Also the results clearly demonstrate the protective and therapeutic effects of GLE against pancreatic carcinogenesis through multiple pathways, with the main molecular targets including iNOS, and miRNAs: $21,34 \mathrm{a}$ and $200 b$.

\section{Acknowledgments}

Mona Yehia, Professor of Histochemistry and cell biology, Medical Research Institute, Histochemistry and cell biology Department, Alexandria University, for her help and cooperation in the histopathology part.

\section{References}

1 Siegel, RL.Cancer statistics, CA Cancer J Clin, 2016, 66,7-30. [PMID: 26742998 DOI: 10.3322/caac.21332]

2 Klöppel,G.;Hruban,R.;Longnecker, D.Tumors of the exocrine pancreas. World Health Organization Classification of TumoursPathology and Genetics of Tumours of the Digestive System IARC Press: Lyon, France, 2000, 219-305. [PMID: 6313514] 
3 Goral, V. Pancreatic cancer: Pathogenesis and diagnosis, Asian Pac J Cancer Prev 2015, 16,5619-5624. [PMID: 26320426 DOI: 10.7314/apjcp.2015.16.14.5619]

4 De La Cruz.; Young, AP.; Ruffin, I. Diagnosis and management of pancreatic cancer, American family physician, 2014, 89(8).

5 Torres, MP.;Rachagani, S.;Purohit, V. Graviola: a novel promising natural-derived drug that inhibits tumorigenicity and metastasis of pancreatic cancer cells in vitro and in vivo through altering cell metabolism, Cancer letters 2012, 323(1),29-40. DOI: 10.1016/j.canlet.2012.03.031

6 Jansen, RJ.; Robinson, DP.; Stolzenberg-Solomon, RZ. Fruit and vegetable consumption is inversely associated with having pancreatic cancer, Cancer Causes Control 2011, 22(12),1613-1625. DOI: 10.1007/s10552-0119838-0

7 Zhang, J.; Go, VLW.; High fat diet, lipid peroxidation, and pancreatic carcinogenesis, 1996, Springer. DOI:doi.org/10.1007/978-14613-1151-5_13

8 Z'graggen, K.; Warshaw, AL.; Werner, J.; Promoting effect of a high-fat/high-protein diet in DMBA-induced ductal pancreatic cancer in rats, Ann surg, 2001, 233(5),688-695. [PMID: 11323507]

9 Longnecker, D. Experimental pancreatic cancer: role of species, sex and diet, Bulletin du cancer, 1989, 77(1)27-37. [PMID:2317574]

10 Kazakoff, K.; Cardesa, T.; Liu, J. Effects of voluntary physical exercise on high-fat dietpromoted pancreatic carcinogenesis in the hamster model, Nutrition and cancer 1996 26(3):265-79. $10.1080 / 01635589609514483$

11 Jimenez, RE.; Z' graggen, K.; Hartwig, W. Immunohistochemical characterization of pancreatic tumors induced by dimethylbenzanthracene in rats, The American journal of pathology, 1999, 154(4), 1223-1229. DOI: $10.1016 /$ S0002-9440(10)65374-6

12 Granger, J.; Remick, D. Acute pancreatitis: models, markers, and mediators, Shock, 2005, 24, 45-51. [PMID: 16374372 DOI: 10.1097/01.shk.0000191413.94461.b0]

13 Zhang, X.; Li, Y.; Ji, Y. Pancreatic Carcinoma with the First Symptom of Acute Pancreatitis: MRI Findings and Clinical Features, Pancreat Disord Ther, 2014. DOI: $\underline{10.4172 / 2165-}$ 7092.1000132
14 Arrigo, A-P. Gene expression and the thiol redox state, Free Radical Biology and Medicine, 1999, 27(9), 936-944. DOI: 10.1016/s0891-5849(99)00175-6

15 Wu, Y.;Zhou, BP. TNF-alpha/NF-kappaB/Snail pathway in cancer cell migration and invasion, Br J Cancer, 2010, 102(4), 639-644. DOI: 10.1038/sj.bjc.6605530

16 Liptay, S.; Weber, CK.; Ludwig, L. Mitogenic and antiapoptotic role of constitutive NF-kappa B Rel activity in pancreatic cancer, International Journal of Cancer, 2003, 105(6),735-746. DOI: 10.1002/ijc.11081

17 Kumari, N.; Dwarakanath, B.; Das, A. Role of interleukin-6 in cancer progression and therapeutic resistance, Tumor Biol, 2016, 37(9),11553-11572. [PMID: 27260630 DOI: 10.1007/s13277-016-5098-7]

18 Wang, T.; Zhang, X.; Li, JJ. The role of NFkappa $\mathrm{B}$ in the regulation of cell stress responses,

International immunopharmacology, 2002, 2(11),15091520. [PMID: 12433052]

19 Fisher, DT.; Appenheimer, MM.; Evans, SS. The two faces of IL-6 in the tumor microenvironment, Semin Immunol, 2014, 38 -47. DOI: 10.1016/i.smim.2014.01.008

20 Neurath, MF.; Finotto, S. IL-6 signaling in autoimmunity, chronic inflammation and inflammation-associated cancer, Cytokine growth factor Rev, 2011, 22(2),83-89. DOI: 10.1016/j.cytogfr.2011.02.003

21 Heinrich, PC.; Behrmann, I.; Serge, H.; Principles of interleukin (IL)-6-type cytokine signalling and its regulation, Biochemical journal, 2003, 374(1), 1-20. DOI: 10.1042/BJ20030407

22 Ricciardi, M.; Zanotto, M.; Malpeli. G. Epithelial-to-mesenchymal transition (EMT) induced by inflammatory priming elicits mesenchymal stromal cell-like immunemodulatory properties in cancer cells, $\boldsymbol{B r} \boldsymbol{J}$ Cancer, 2015, 112(6), 1067-1075. DOI: 10.1038/bjc.2015.29

23 Carpenter, RL.; Lo, H-W. STAT3 target genes relevant to human cancers, Cancers, 2014, 6(2), 897-925. DOI: $10.3390 /$ cancers6020897

24 Park, MH.; Hong, JT. Roles of NF-kappa B in cancer and inflammatory diseases and their therapeutic approaches, Cells, 2016, 5(2),15. DOI: $10.3390 /$ cells5020015

25 Tucker, ON.; Dannenberg, AJ.; Yang, EK. Cyclooxygenase-2 expression is up-regulated 
in human pancreatic cancer, Cancer Res, 1999, 59(5),987-990. [PMID: 10070951]

26 Kiyohara, H., Egami, H.; Kako, H.; Immunohistochemical localization of group II phospholipase A2 in human pancreatic carcinomas, Int J Pancreatol, 1993, 13(1),4957. DOI: $10.1007 / \mathrm{BF} 02795199$

27 Hanahan, D.; Folkman, J. Patterns and emerging mechanisms of the angiogenic switch during tumorigenesis, cell, 1996, 86(3), $353-364$. [PMID: $\quad 8756718$ DOI: $10.1016 / \mathrm{s} 0092-$ 8674(00)80108-7]

28 Liszka, L,; Pajkak, J.; Golka, D.; Serous neoplasms of the pancreas share many, but not all aspects of their microvascular and angiogenic profile with low-grade clear cell renal cell carcinomas, Pathol Res Pract, 2014, 210(12), 901-908. [PMID: 21763962]

29 Costache, M.; Ioana, M.; Iordache, S. VEGF expression in pancreatic cancer and other malignancies: a review of the literature, Rom J Intern Med, 2015, 53(3), 199-208. [PMID: 26710495 DOI: $10.1515 / \mathrm{rjim}-2015-0027]$

30 Ferreiro, C.R.; JLCBDS. Influence of hypoxia on nitric oxide synthase activity and gene expression in children with congenital heart disease novel patho-physiological adaptive mechanism, Circulation, 2001, 103, 2272-2276. DOI: $10.1161 / 01 . c i r .103 .18 .2272$

31 Eguchi, Y; Shimizu, S.; Tsujimoto, Y.; Intracellular ATP levels determine cell death fate by apoptosis or necrosis, Can Res, 1997, 57(10), 1835-1840. [PMID: 9157970]

32 Mankan , A.K. NF-kappa B regulation: the nuclear response, J Cell Mol Med, 2009, 13, 631-643. $\quad$ DOI: $\quad 10.1111 / \mathrm{j} .1582-$ 4934.2009.00632.x

33 A.Pautz, J.Art. Regulation of the expression of inducible nitric oxide synthase, Nitric Oxide: BiolChem, 2010, 23,75-93. [PMID: 20438856 DOI: $10.1016 /$ j.niox.2010.04.007]

34 Niu, J.; Shi, Y.; Tan, G. DNA damage induces NF-kappa B-dependent microRNA-21 upregulation and promotes breast cancer cell invasion, J Biol Chem, 2012, 287(26), 2178321795. DOI: $10.1074 /$ jbc.M112.355495

35 Baud, V.; Karin, M. Is NF-kappa B a good target for cancer therapy? Hopes and pitfalls, Nat Rev Drug Discov, 2009, 8(1), 33-40. DOI: $10.1038 / \mathrm{nrd} 2781$

36 Iliopoulos, D.; Hirsch, HA.;Struhl, K.; An epigenetic switch involving NF-kappa B, Lin28, Let-7 MicroRNA, and IL6 links inflammation to cell transformation, Cell, 2009, 139(4), 693706. DOI: $10.1016 /$ j.cell.2009.10.014

$37 \mathrm{Yu}$, J.;Ohuchida, K.; Mizumoto, K;. MicroRNA, hsa-miR-200c, is an independent prognostic factor in pancreatic cancer and its upregulation inhibits pancreatic cancer invasion but increases cell proliferation, Mol Cancer, 2010, 9(1), 169. [PMID: 20579395 PMCID: PMC2909980 DOI: 10.1186/1476-4598-9-169]

38 Du, Y.; Liu, M.; Gao, J. Aberrant microRNAs expression patterns in pancreatic cancer and their clinical translation, Cancer Biotherapy and Radiopharmaceuticals, 2013, 28(5),361$369 . \quad$ [PMID: 23621126 DOI: 10.1089/cbr.2012.1389]

39 Chang, T-C.; Wentzel, EA.; Kent, OA. Transactivation of miR-34a by p53 broadly influences gene expression and promotes apoptosis, Molecular cell, 2007, 26(5), 745752. [PMID: 17540599 PMCID: PMC1939978 DOI: $10.1016 /$ j.molcel.2007.05.010]

40 Ji, Q.; Hao, X.; Zhang, M. MicroRNA miR-34 inhibits human pancreatic cancer tumorinitiating cells, PloS one, 2009, 4(8):e6816. [PMID: 19714243 PMCID: PMC2729376 DOI: 10.1371/journal.pone.0006816]

41 Yoshimasu, T.; Maebeya, S.; Suzuma, T. Disappearance curves for tumor markers after resection of intrathoracic malignancies, Int $\boldsymbol{J}$ Biol Markers, 1999, 14(2),99-105. [PMID: 10399629]

42 Taylor, L. Technical Data Report for Graviola: Annona Muricata, Sage Press Austin, 2002, 10,1. DOI:10.4236/abcr.2014.33012

43 Hamid, RA.; Foong, CP.; Ahmad, Z. Antinociceptive and anti-ulcerogenic activities of the ethanolic extract of Annona muricata leaf, Rev Bras Farmacog, 2012, 22(3), 630641. DOI:doi.org/10.1590/S0102695X2012005000001

44 Minari, J.; Okeke, U.; Chemopreventive effect of Annona muricata on DMBA-induced cell proliferation in the breast tissues of female albino mice, Egypt J Med Hum Genet, 2014, 15(4),327-4.

DOI:doi.org/10.1016/j.ejmhg.2014.05.001

45 Hamizah, S.; Roslida, A.; Fezah, O. Chemopreventive potential of Annona muricata L leaves on chemically-induced skin papillomagenesis in mice, Asian Pac J Cancer Prev, 2012, 13(6), 2533-2539. [PMID: 22938417 DOI: 10.7314/apjcp.2012.13.6.2533] 